\title{
GEOMETRIC RENORMALIZATION BELOW THE GROUND STATE
}

\author{
PAUL SMITH
}

\begin{abstract}
The caloric gauge was introduced in 23 by Tao with studying large data energy-critical wave maps mapping from $\mathbf{R}^{2+1}$ to hyperbolic space $\mathbf{H}^{m}$ in view. In [1] Bejenaru, Ionescu, Kenig, and Tataru adapted the caloric gauge to the setting of Schrödinger maps from $\mathbf{R}^{d+1}$ to the standard sphere $S^{2} \hookrightarrow \mathbf{R}^{3}$ with initial data small in the critical Sobolev norm. Here we develop the caloric gauge in a bounded geometry setting with a construction valid up to the ground state energy.
\end{abstract}

1. Introduction

2. Notation and basic estimates 6

3. Geometry 7

4. Local theory and continuation 11

5. Local energy estimates 21

6. Minimal blowup solutions $\quad 23$

7. The caloric gauge $\quad 26$

Appendix A. Heat flow into noncompact manifolds

Appendix B. An energy space $\quad 36$

References $\quad 38$

Contents

\section{INTRODUCTION}

Certain geometric PDE, such as wave maps and Schrödinger maps, enjoy the property of gauge invariance at the level of the tangent bundle. The particular gauge selection one makes plays a crucial role in the analysis of the PDE in question. The Coulomb gauge is a well-known, classical choice well-suited for many problems. However, when analyzing energy-critical wave maps and Schrödinger maps, for instance, one finds that the Coulomb gauge leaves something to be desired. Tao proposed an alternative (see [23]), the caloric gauge, which, in particular, handles high $\times$ high $\rightarrow$ low frequency interactions much more favorably than does the Coulomb gauge. Up until now, the caloric gauge was available only in very restricted settings, i.e., for target manifolds of constant negative curvature or for initial data that is suitably small in an appropriate sense. It is the purpose of this paper to provide a construction of the caloric gauge valid in a very general setting. 
The key tool behind the caloric gauge construction is yet another geometric PDE: the harmonic map heat flow. Harmonic maps and their heat flows have been intensely studied, and their literature is extensive. The pioneering work on harmonic map heat flow is due to Eells and Sampson [5]. Our work leans most heavily upon the original results of Struwe [20, though we have striven to make this paper largely self-contained in its presentation. One major exception to this is found in $\$ 6$, where we cite a major result of [20]. The other major exception is found in Appendix A, where we cite a result of $\mathrm{Li}$ and Tam [8] in order to justify moving from a compact setting to a noncompact setting. The contributions this paper makes to the theory of the harmonic map heat flow are of a technical and analytic nature. While no new qualitative results are shown, several hard quantitative estimates established do appear to be new; these quantitative estimates play an important role both in the caloric gauge construction itself and in establishing the appealing quantitative estimates that the caloric gauge enjoys.

We mention here some of the developments in other directions of the theory of harmonic maps and harmonic map heat flow. Results establishing in certain settings the nonexistence of smooth nontrivial harmonic maps are found in Eells-Wood [4] and Lemaire [7]. Regularity results originate in Morrey [14. See Schoen-Uhlenbeck [16] for a compactness property and boundary regularity. Uniform estimates on derivatives of stationary harmonic maps are derived in [9]. See Struwe [20] for global weak solutions and bubbling, and Topping [28, 29, 30, 31, 32, 33], for instance, for sophisticated extensions and refinements along these lines. For global existence of weak heat flows, see Chen [2], Chen-Struwe [3]. Recent global heat flow results for rough initial data are due to Koch-Lamm [6]. See Lin-Wang [10] for work on convergence of sequences of heat flows. For the consideration of noncompact target manifolds, see Mitteau [13, Li-Tam 8], and the references therein. The book [1] by Lin-Wang provides an extensive (though not exhaustive) survey.

Let $\mathcal{M}$ denote an $m$-dimensional Riemannian manifold with metric $h$ and let $\mathbf{R}^{+}:=[0,+\infty)$. A function $\phi: \mathbf{R}^{+} \times \mathbf{R}^{2} \rightarrow \mathcal{M}$ satisfying

$$
\partial_{s} \phi(s, x)=\left(\phi^{*} \nabla\right){ }_{j} \partial_{j} \phi(s, x),
$$

where $\phi^{*} \nabla$ is the pullback by $\phi$ of the Riemannian connection $\nabla$ on $\mathcal{M}$, we call a harmonic map heat flow. Here we parametrize $\mathbf{R}^{+} \times \mathbf{R}^{2}$ by $\left(s, x_{1}, x_{2}\right)$, interpreting $s$ as a time variable and implicitly summing repeated Roman indices over the spatial variables so that $j$ ranges over 1,2 . We usually abbreviate "harmonic map heat flow" to just heat flow. A map defined and satisfying (1.1) on a time subinterval $I$ of $\mathbf{R}^{+}$we also call a heat flow, though sometimes we instead refer to such maps as partial heat flows, particularly when we wish to emphasize that a priori such maps do not admit suitably defined extensions from $I$ to all of $\mathbf{R}^{+}$. 
We note that (1.1) is invariant with respect to the following translational and dilational symmetries:

$$
\begin{aligned}
\phi(s, x) & \mapsto \phi\left(s, x-x_{0}\right) & x_{0} \in \mathbf{R}^{2}, \\
\phi(s, x) & \mapsto \phi\left(\frac{s}{\lambda^{2}}, \frac{x}{\lambda}\right) & \lambda>0 .
\end{aligned}
$$

We assume throughout that $\mathcal{M}$ is a smooth manifold and has bounded geometry, meaning that the injectivity radius of $\mathcal{M}$ is bounded from below and that the associated Riemannian curvature tensor $\mathcal{R}$ and its covariant derivatives are bounded uniformly on $\mathcal{M}$ (e.g., see [34, Chapter 7] for discussion and additional references).

While the vast majority of our arguments are intrinsic, we shall appeal to extrinsic arguments in order to establish local existence. To avoid distracting technicalities, we therefore make the additional working assumption that $\mathcal{M}$ is compact and without boundary (i.e., a closed manifold). We make implicit use of compactness in $\$ 6$. In Appendix $\mathrm{A}$ we show how to remove the technical compactness assumption and include a list of all places where we had made use of it; one motivation for doing so is to capture within our framework the case where $\mathcal{M}$ is a hyperbolic space $\mathbf{H}^{m}$. As the additional arguments we present essentially reduce the more general setting to the compact case, we therefore have a unified approach for constructing the caloric gauge, valid for all bounded geometry manifolds $\mathcal{M}$.

By the Nash Embedding Theorem, $\mathcal{M}$ admits a smooth isometric embedding $\iota: \mathcal{M} \hookrightarrow \mathbf{R}^{n}$ into a Euclidean space of (typically higher) dimension $n$. Compactness ensures boundedness of the second fundamental form and closely related shape operator, which, informally speaking, describe how serpentine the embedding is. The extrinsic formulation of (1.1) that $\iota$ gives rise to and that we discuss below is used to establish local existence and uniqueness for (1.1) and to derive a blowup criterion. All other arguments and in fact the blowup criterion itself admit intrinsic formulations. Consequently, all of our main conclusions are independent of the particular value of $n$ or choice of embedding $\iota$.

Let $T \mathcal{M}$ denote the tangent bundle of $\mathcal{M}$ and, using the embedding $\iota$, define $N \mathcal{M}$ to be its normal bundle in $\mathbf{R}^{n}$. The second fundamental form $\Pi$ of $\mathcal{M}$ is a symmetric bilinear form $\Pi: T \mathcal{M} \times T \mathcal{M} \rightarrow N \mathcal{M}$ satisfying

$$
\langle\Pi(X, Y), N\rangle=\left\langle\partial_{X} N, Y\right\rangle
$$

for vector fields $X, Y$ in $T \mathcal{M}$ and $N$ in $N \mathcal{M}$, according to the sign convention we adopt. The heat flow equation (1.1) then admits the extrinsic formulation

$$
\partial_{s} \phi=\Delta \phi+\Pi(\phi)\left(\partial_{x} \phi, \partial_{x} \phi\right),
$$

where here we have identified $\iota \circ \phi$ with $\phi$ just as we shall do in the sequel when convenient and without further comment. 
We assume we are given Cauchy initial data to (1.1) so that $\phi$ is specified at an initial time $s_{0}$, which we usually take to be zero:

$$
\phi(0, x)=\phi_{0}(x) .
$$

Throughout we require that $\phi_{0}$ be classical initial data, by which we mean that $\iota \circ \phi_{0}$ of $\phi_{0} \in C_{x}^{\infty}\left(\mathbf{R}^{2} \rightarrow \mathcal{M}\right)$ differs from a constant $\phi_{0}(\infty) \in \mathcal{M} \hookrightarrow_{\iota} \mathbf{R}^{n}$ by some Schwartz function $\mathbf{R}^{2} \rightarrow \mathbf{R}^{n}$. That this definition is independent of the choice of embedding $\iota$ can be checked by examining $\tilde{\iota} \circ \iota^{-1} \circ\left(\iota \circ \phi_{0}\right)$ for some other choice of smooth isometric embedding $\tilde{\iota}$. A smooth function $\phi: \mathbf{R}^{2} \rightarrow \mathcal{M}$ differing from some $\phi(\infty) \in \mathcal{M}$ by a Schwartz function we also call Schwartz with respect to $\phi(\infty)$. Schwartz functions admit an intrinsic definition (entirely equivalent in the case of compact $\mathcal{M}$ ) in terms of a base point $p \in \mathcal{M}$ and the connection $\nabla$, though we postpone further discussion of this to Appendix A.

Under these assumptions there exists at least for short time a unique smooth heat flow $\phi$ that satisfies (1.1) and (1.5) in the classical sense. In fact, global existence and uniqueness hold in this sense (see [20]) provided the initial data $\phi_{0}$ has energy

$$
E_{0}:=E\left(\phi_{0}\right):=\frac{1}{2} \int_{\mathbf{R}^{2}}\left|\partial_{x} \phi_{0}\right|_{\phi^{*} h}^{2} d x
$$

below some $\mathcal{M}$-dependent threshold which we call $E_{\text {crit }}$. In particular, $E_{\text {crit }}$ is equal to the least energy that may be carried by a nontrivial finite-energy harmonic map mapping $\mathbf{R}^{2} \rightarrow \mathcal{M}$ and is set equal to $\infty$ if $\mathcal{M}$ admits no such maps. We call this threshold $E_{\text {crit }}$ the ground state energy. That such a threshold exists (or can be taken to be $\infty$ ) follows easily from a certain a priori gradient estimate; see [11, Chapter 6] for details. Note that stationary solutions to (1.1) may be identified with harmonic maps. The aptly-named harmonic map heat flow equation (1.1) is the downward $L^{2}$ gradient flow associated with the energy functional

$$
\phi \mapsto \frac{1}{2} \int_{\mathbf{R}^{2}}\left|\partial_{x} \phi\right|^{2} d x
$$

and therefore heuristically may be thought to asymptotically evolve finite energy maps into solutions of the harmonic map equation

$$
\left(\phi^{*} \nabla\right)_{j} \partial_{j} \phi(x)=0 \text {. }
$$

In $\oint 2$ we review some notation and basic estimates. The technical heart lies in establishing for various energy quantities associated to $\phi$ bounds depending only upon the initial energy $E_{0}$. As the energy functional given by (1.6) is invariant with respect to the scaling (1.3) enjoyed by (1.1), these bounds are both energy-critical and critical with respect to the heat flow equation. In addition to recalling some constructions from Riemannian geometry, in $\$ 3$ we prove a set of Bochner-Weitzenböck estimates that play 
a key role in establishing energy bounds, which we then proceed to establish in a direct manner for small energies in $\$$. In $\$ 6$ we use an induction on energy argument, formulated in terms of "minimal blow-up solutions", along with concentration compactness to extend the energy estimates to all energies below the ground state threshold $E_{\text {crit }}$. In particular, we show that should these estimates not hold for all maps $\phi$ with energy below $E_{\text {crit }}$, then by exploiting the symmetries (1.2) and (1.3) one may extract a nontrivial harmonic map of energy strictly less than $E_{\text {crit }}$, thereby contradicting the definition of $E_{\text {crit }}$. The main estimates used in extracting the harmonic map and proving convergence originate in the work of Struwe and are proven intrinsically in $\$ 5$; see [20, 21].

In $\$ 7$ we introduce the notion of a gauge and in particular the caloric gauge. The caloric gauge was introduced by Tao in 23 in the context of wave maps. In 22] Tao suggested that the caloric gauge could be effectively applied to the study of Schrödinger maps. The caloric gauge has been successfully used in the study of global wellposedness for wave maps and Schrödinger maps: See Tao's application to wave maps in [25-27] and the application of Bejenaru, Ionescu, Kenig, and Tataru to Schrödinger maps in [1].

To see how the caloric gauge compares to the Coulomb gauge, see [23], 22] and also [1, §2]. For a general discussion on gauges and a comparison of several different gauges, see [24, Chapter 6].

Up until now, in the presence of positive curvature the caloric gauge has only been available under the restrictive assumption of small energy. 1 We offer a construction valid up to the ground state energy $E_{\text {crit }}$. In particular, our main results are summarized as follows:

- Theorem 6.2: Given classical initial data $\phi_{0}$ with $E\left(\phi_{0}\right)<E_{\text {crit }}$, we establish global existence and uniqueness of a smooth heat flow $\phi$ as well as a host of quantitative energy estimates.

- Theorem 7.2: Given classical initial data $\phi_{0}$ with $E\left(\phi_{0}\right)<E_{\text {crit }}$, we establish existence and uniqueness (up to a choice of boundary frame) of the caloric gauge $e \in \Gamma\left(\operatorname{Frame}\left(\phi^{*} T \mathcal{M}\right)\right)$. In the remainder of 97 , we use Theorem 6.2 to prove quantitative estimates on various gauge components.

- Theorem 7.6: We show that the static caloric gauge construction of Theorem 7.2 is easily adapted to the dynamic setting.

For applications of these results to the study of Schrödinger maps, we refer the reader to the work of the author in [18, 17].

\footnotetext{
${ }^{1}$ For maps mapping from a higher dimensional base space, the appropriate assumption is small critical Sobolev norm. See for instance the application to Schrödinger maps in [1. In this paper we only consider two spatial dimensions.
} 


\section{Notation AND BASiC ESTimates}

We use $f \lesssim g$ to denote the estimate $|f| \leq C|g|$ for an absolute constant $C>0$. To indicate dependence of the constant upon parameters (which, for instance, can include functions), we use subscripts, e.g. $f \lesssim_{k} g$. As an equivalent alternative we write $f=O(g)$ (or, with subscripts, $f=O_{k}(g)$ ) to denote $|f| \leq C|g|$.

In addition to the usual $L_{x}^{p}$ spaces, we employ the Banach spaces $C_{x}^{k}$ of $k$-times continuously differentiable functions, $k=0,1,2, \ldots$, equipped with the norm

$$
\|u\|_{C_{x}^{k}\left(\mathbf{R}^{2}\right)}:=\sup _{0 \leq j \leq k} \sup _{x \in \mathbf{R}^{2}}\left|\partial_{x}^{j} u(x)\right|
$$

and corresponding seminorm

$$
\|u\|_{\dot{C}_{x}^{k}\left(\mathbf{R}^{2}\right)}:=\sup _{x \in \mathbf{R}^{2}}\left|\partial_{x}^{k} u(x)\right|,
$$

where we use $\partial_{x}=\left(\partial_{x_{1}}, \partial_{x_{2}}\right)$ to denote the gradient operator, as throughout " $\nabla$ " will stand for the Riemannian connection on $\mathcal{M}$. On occasion we use the usual Sobolev spaces $W_{x}^{s, p}, H_{x}^{s}:=W_{x}^{s, 2}$, their homogeneous counterparts $\dot{W}_{x}^{s, p}, \dot{H}_{x}^{s}$, and their localized versions $W_{x, \text { loc }}^{s, p}, H_{x, \text { loc }}^{s}$.

We record the following special cases of the well-known Gagliardo-Nirenberg inequality for later use:

$$
\begin{aligned}
\|u\|_{L_{x}^{\infty}\left(\mathbf{R}^{2}\right)} & \lesssim\|u\|_{L_{x}^{2}\left(\mathbf{R}^{2}\right)}^{1 / 2}\left\|\partial_{x}^{2} u\right\|_{L_{x}^{2}\left(\mathbf{R}^{2}\right)}^{1 / 2} \\
\|u\|_{L_{x}^{\infty}\left(\mathbf{R}^{2}\right)} & \lesssim\|u\|_{L_{x}^{2}\left(\mathbf{R}^{2}\right)}^{1 / 3}\left\|\partial_{x} u\right\|_{L_{x}^{4}\left(\mathbf{R}^{2}\right)}^{2 / 3} \\
\|u\|_{L_{x}^{4}\left(\mathbf{R}^{2}\right)} & \lesssim\|u\|_{L_{x}^{2}\left(\mathbf{R}^{2}\right)}^{1 / 2}\left\|\partial_{x} u\right\|_{L_{x}^{2}\left(\mathbf{R}^{2}\right)}^{1 / 2}
\end{aligned}
$$

Throughout $\Delta:=\partial_{x_{1}}^{2}+\partial_{x_{2}}^{2}$ stands for the usual spatial Laplacian on $\mathbf{R}^{2}$. The heat kernel is defined by

$$
e^{s \Delta} u(x):=\frac{1}{4 \pi s} \int_{\mathbf{R}^{2}} e^{-\frac{|x-y|^{2}}{4 s}} u(y) d y
$$

By Young's inequality the parabolic regularity estimate

$$
\left\|\partial_{x}^{k} e^{s \Delta} u\right\|_{L_{x}^{q}\left(\mathbf{R}^{2}\right)} \lesssim_{p, q, k} s^{\frac{1}{q}-\frac{1}{p}-\frac{k}{2}}\|u\|_{L_{x}^{p}\left(\mathbf{R}^{2}\right)}
$$

holds for all $s>0, k \geq 0$, and $1 \leq p \leq q \leq \infty$. In particular,

$$
\left\|e^{s \Delta} u\right\|_{\dot{C}_{x}^{1}\left(\mathbf{R}^{2}\right)} \lesssim s^{-1 / 2}\|u\|_{C_{x}^{0}\left(\mathbf{R}^{2}\right)}
$$

and

$$
\left\|e^{s \Delta} u\right\|_{C_{x}^{1}\left(\mathbf{R}^{2}\right)} \lesssim\left(1+s^{-1 / 2}\right)\|u\|_{C_{x}^{0}\left(\mathbf{R}^{2}\right)} .
$$

We shall frequently make use of the following more refined variant of (2.5): 
Lemma 2.1. (Integrated parabolic regularity). For any $u \in L_{x}^{2}\left(\mathbf{R}^{2}\right)$ and $2<p \leq \infty$ we have

$$
\int_{0}^{\infty} s^{-2 / p}\left\|e^{s \Delta} u\right\|_{L_{x}^{p}\left(\mathbf{R}^{2}\right)}^{2} d s \lesssim p\|u\|_{L_{x}^{2}\left(\mathbf{R}^{2}\right)}^{2} .
$$

Proof. The lemma follows from the $T T^{*}$ method. See [26, Lemma 2.5] for details.

Because (2.8) is analogous to certain Strichartz estimates, standard in the study of dispersive PDE, what we call "integrated parabolic regularity" [26] dubs a "parabolic Strichartz estimate".

We also note Duhamel's formula

$$
u\left(s_{1}\right)=e^{\left(s_{1}-s_{0}\right) \Delta} u\left(s_{0}\right)+\int_{s_{0}}^{s_{1}} e^{\left(s_{1}-s\right) \Delta}\left(\partial_{s} u-\Delta u\right)(s) d s,
$$

valid for any continuous map $s \mapsto u(s)$ from the interval $\left[s_{0}, s_{1}\right]$ to the space of tempered distributions on $\mathbf{R}^{2}$, which we may take to be either scalar valued or vector valued.

\section{Geometry}

Recall $\mathcal{M}$ is an $m$-dimensional Riemannian manifold with metric $h$. There then exists a unique Riemannian connection $\nabla$ on $\mathcal{M}$ satisfying the following four characterizing properties. Given any smooth vector fields $X, Y, Z$ on $\mathcal{M}$ and smooth real-valued function $f$ on $\mathcal{M}$, the Riemannian connection $\nabla$ is linear

$$
\nabla_{f X} Y=f \nabla_{X} Y
$$

satisfies the Leibniz rule

$$
\nabla_{X}(f Y)=\left(\partial_{X} f\right) Y+f \nabla_{X} Y
$$

respects the metric

$$
\partial_{X} h(Y, Z)=h\left(\nabla_{X} Y, Z\right)+h\left(Y, \nabla_{X} Z\right),
$$

and is torsion-free

$$
\nabla_{X} Y-\nabla_{Y} X=[X, Y]
$$

Here $[X, Y]$ denotes the Lie bracket of $X$ and $Y$. The Riemannian curvature tensor $\mathcal{R}$ on $\mathcal{M}$ is a $(1,3)$-tensor defined by

$$
\mathcal{R}(X, Y) Z=\nabla_{X} \nabla_{Y} Z-\nabla_{Y} \nabla_{X} Z-\nabla_{[X, Y]} Z .
$$

We caution the reader that oftentimes $\mathcal{R}$ is defined with the sign convention opposite to the one we adopt. Via the metric $h$ on $\mathcal{M}$ the tensor $\mathcal{R}$ is sometimes viewed as a $(0,4)$-tensor. The curvature tensor enjoys several other well-known properties, e.g., symmetries, though we shall not make 
explicit use of these in this paper. A good general introduction and reference is [15, Chapter 2].

Recall $\mathcal{M}$ is isometrically embedded by some $\iota$ in $\mathbf{R}^{n}$. By definition, the pullback via $\iota^{*}$ of the metric on $\iota(\mathcal{M})$ inherited from the ambient space $\mathbf{R}^{n}$ coincides with the intrinsic metric $h$. Compactness implies that the second fundamental form $\Pi$ and its derivatives are bounded uniformly.

Let $\phi: \mathbf{R}^{d} \rightarrow \mathcal{M}$ be a smooth map from the vector space $\mathbf{R}^{d}$ to the manifold $\mathcal{M}$. We may then pull back the tangent bundle $T \mathcal{M}$ by $\phi$ to a vector bundle $\phi^{*}(T \mathcal{M})$ over $\mathbf{R}^{d}$. A smooth section $\gamma: \mathbf{R}^{d} \rightarrow \phi^{*}(T \mathcal{M})$ of this vector bundle is a smooth assignment of a tangent vector $\gamma(x) \in T_{\phi(x)} \mathcal{M}$ to each $x \in \mathbf{R}^{d}$. We may also pull back the connection $\nabla$ on $\mathcal{M}$ to a connection $\phi^{*} \nabla$ on $\mathbf{R}^{d}$. Let $\partial_{1}, \ldots, \partial_{d}$ denote the standard coordinate vector fields on $\mathbf{R}^{d}$ and let $\gamma, \gamma^{\prime}$ be smooth sections of $\phi^{*}(T \mathcal{M})$. Then from (3.2) we derive the Leibniz rule

$$
\partial_{i}\left\langle\gamma, \gamma^{\prime}\right\rangle_{\phi^{*} h}=\left\langle\left(\phi^{*} \nabla\right)_{i} \gamma, \gamma^{\prime}\right\rangle_{\phi^{*} h}+\left\langle\gamma,\left(\phi^{*} \nabla\right)_{i} \gamma^{\prime}\right\rangle_{\phi^{*} h},
$$

and from (3.4) the zero-torsion property

$$
\left(\phi^{*} \nabla\right)_{i} \partial_{j} \gamma=\left(\phi^{*} \nabla\right)_{j} \partial_{i} \gamma
$$

Note that these constructions readily apply to $I \times \mathbf{R}^{d}$ in place of $\mathbf{R}^{d}$ when we take $I$ to be a connected interval.

For each positive integer $k$ define the energy densities $\mathbf{e}_{k}$ of a map $\phi$ to be

$$
\begin{aligned}
\mathbf{e}_{k} & :=\left|\left(\phi^{*} \nabla\right)_{x}^{k-1} \partial_{x} \phi\right|_{\phi^{*} h}^{2} \\
& :=\left\langle\left(\phi^{*} \nabla\right)_{j_{1}} \ldots\left(\phi^{*} \nabla\right)_{j_{k-1}} \partial_{j_{k}} \phi,\left(\phi^{*} \nabla\right)_{j_{1}} \ldots\left(\phi^{*} \nabla\right)_{j_{k-1}} \partial_{j_{k}} \phi\right\rangle_{\phi^{*} h},
\end{aligned}
$$

where $j_{1}, \ldots, j_{k}$ are summed over 1,2 .

Lemma 3.1. (Bochner-Weitzenböck identities). Let $\phi$ be a heat flow. Then for each $k \geq 1$ we have

$$
\partial_{s} \mathbf{e}_{k}=\Delta \mathbf{e}_{k}-2 \mathbf{e}_{k+1}+N_{k},
$$

where $N_{k}$ is a linear combination of $O_{k}(1)$ terms of the form

$$
T(\phi)\left(X_{1}, \ldots, X_{j}, Y\right),
$$

where $T$ is a tensor with bounded covariant derivatives (bounded on $\mathcal{M}$ ), $j \in\{3,4, \ldots, k+2\}, Y$ is an iterated spatial covariant derivative of $\phi$ of order $k$, and each $X_{\ell}$ is an iterated spatial covariant derivative of $\phi$ of positive order so that for each $j$ the sum of orders of derivatives (associated to the $X_{\ell}$ ) equals $k+2$. Moreover, $N_{k}$ satisfies the bound

$$
\left|N_{k}\right| \lesssim_{k} \sum_{j=3}^{k+2} \sum_{a_{1}+\cdots+a_{j}=k+2}\left(\mathbf{e}_{a_{1}} \cdots \mathbf{e}_{a_{j}} \mathbf{e}_{k}\right)^{1 / 2} .
$$


Proof. We first prove the $k=1$ case, which plays a privileged role throughout the sequel. By the Leibnitz rule (3.5) and zero-torsion property (3.6) it holds that

$$
\begin{aligned}
\partial_{s} \mathbf{e}_{1} & =\partial_{s}\left\langle\partial_{i} \phi, \partial_{i} \phi\right\rangle_{\phi^{*} h} \\
& =2\left\langle\left(\phi^{*} \nabla\right)_{s} \partial_{i} \phi, \partial_{i} \phi\right\rangle_{\phi^{*} h} \\
& =2\left\langle\left(\phi^{*} \nabla\right)_{i} \partial_{s} \phi, \partial_{i} \phi\right\rangle_{\phi^{*} h} .
\end{aligned}
$$

Curvature arises in commuting the covariant derivatives so that

$$
\begin{aligned}
\left(\phi^{*} \nabla\right)_{i} \partial_{s} \phi & =\left(\phi^{*} \nabla\right)_{i}\left(\phi^{*} \nabla\right)_{j} \partial_{j} \phi \\
& =\left(\phi^{*} \nabla\right)_{j}\left(\phi^{*} \nabla\right)_{i} \partial_{j} \phi+\mathcal{R}(\phi)\left(\partial_{i} \phi, \partial_{j} \phi\right) \partial_{j} \phi \\
& =\left(\phi^{*} \nabla\right)_{j}\left(\phi^{*} \nabla\right)_{j} \partial_{i} \phi+\mathcal{R}(\phi)\left(\partial_{i} \phi, \partial_{j} \phi\right) \partial_{j} \phi,
\end{aligned}
$$

where the last line follows from (3.6). From (3.5) we also obtain

$$
\Delta \mathbf{e}_{1}=2 \mathbf{e}_{2}+2\left\langle\left(\phi^{*} \nabla\right)_{j}\left(\phi^{*} \nabla\right)_{j} \partial_{i} \phi, \partial_{i} \phi\right\rangle_{\phi^{*} h} .
$$

It follows from (3.9)-(3.11) that

$$
\partial_{s} \mathbf{e}_{1}=\Delta \mathbf{e}_{1}-2 \mathbf{e}_{2}+\left\langle\mathcal{R}(\phi)\left(\partial_{i} \phi, \partial_{j} \phi\right) \partial_{j} \phi, \partial_{i} \phi\right\rangle_{\phi^{*} h},
$$

which completes the proof of the $k=1$ case in view of the bounded geometry assumption.

For each $k \geq 1$ and each $k$-index $\mathbf{m}=\left(m_{1}, \ldots, m_{k}\right), m_{\ell} \in\{1,2\}$, set

$$
\mathfrak{c}_{s, \mathbf{m}}^{k}:=\left(\phi^{*} \nabla\right)_{s}\left(\phi^{*} \nabla\right)_{\mathbf{m}}^{k} \phi-\left(\phi^{*} \nabla\right)_{\mathbf{m}}^{k} \partial_{s} \phi
$$

and

$$
\mathfrak{c}_{\mathbf{m}, j}^{k}:=\left(\phi^{*} \nabla\right)_{\mathbf{m}}^{k}\left(\phi^{*} \nabla\right)_{j} \partial_{j} \phi-\left(\phi^{*} \nabla\right)_{j}\left(\phi^{*} \nabla\right)_{j}\left(\phi^{*} \nabla\right)_{\mathbf{m}}^{k} \phi,
$$

where $\left(\phi^{*} \nabla\right)_{\mathrm{m}}^{k} \phi$ stands for

$$
\left(\phi^{*} \nabla\right)_{\tilde{\mathbf{m}}}^{k-1} \partial_{m_{k}} \phi,
$$

with $\tilde{\mathbf{m}}=\left(m_{1}, \ldots, m_{k-1}\right)$ so that $\mathbf{m}=\left(\tilde{\mathbf{m}}, m_{k}\right)$. With an implicit sum over $\{1,2\}^{k}$ on repeated multiindices $\mathbf{m}$, we have the straightforward relations

$$
\partial_{s} \mathbf{e}_{k}=2\left\langle\left(\phi^{*} \nabla\right)_{s}\left(\phi^{*} \nabla\right)_{\mathbf{m}}^{k} \phi,\left(\phi^{*} \nabla\right)_{\mathbf{m}}^{k} \phi\right\rangle_{\phi^{*} h}
$$

and

$$
\Delta \mathbf{e}_{k}=2 \mathbf{e}_{k+1}+2\left\langle\left(\phi^{*} \nabla\right)_{j}\left(\phi^{*} \nabla\right)_{j}\left(\phi^{*} \nabla\right)_{\mathbf{m}}^{k} \phi,\left(\phi^{*} \nabla\right)_{\mathbf{m}}^{k} \phi\right\rangle_{\phi^{*} h},
$$

so that

$$
\partial_{s} \mathbf{e}_{k}-\Delta \mathbf{e}_{k}+2 \mathbf{e}_{k+1}=2\left\langle\mathfrak{c}_{s, \mathbf{m}}^{k}+\mathfrak{c}_{\mathbf{m}, j}^{k},\left(\phi^{*} \nabla\right)_{\mathbf{m}}^{k} \phi\right\rangle_{\phi^{*} h} .
$$

Taking now $\tilde{\mathbf{m}}=\left(m_{2}, \ldots, m_{k}\right)$ so that $\mathbf{m}=\left(m_{1}, \tilde{\mathbf{m}}\right)$, we also have the recursive relations

$$
\mathfrak{c}_{s, \mathbf{m}}^{k}=\sum_{m_{1}=1,2}\left(\phi^{*} \nabla\right)_{m_{1}} \mathfrak{c}_{s, \tilde{\mathbf{m}}}^{k-1}-\sum_{\mathbf{m} \in\{1,2\}^{k}} \mathcal{R}(\phi)\left(\partial_{m_{1}} \phi, \partial_{s} \phi\right)\left(\phi^{*} \nabla\right)_{\tilde{\mathbf{m}}}^{k-1} \phi
$$


and

$$
\begin{aligned}
& \mathfrak{c}_{\mathbf{m}, j}^{k}=\sum_{m_{1}=1,2}\left(\phi^{*} \nabla\right)_{m_{1}} \mathfrak{c}_{\tilde{\mathbf{m}}, j}^{k-1}+\sum_{\mathbf{m} \in\{1,2\}^{k}} {\left[\mathcal{R}(\phi)\left(\partial_{m_{1}} \phi, \partial_{j} \phi\right)\left(\phi^{*} \nabla\right)_{j}\left(\phi^{*} \nabla\right)_{\tilde{\mathbf{m}}}^{k-1} \phi\right.} \\
&\left.+\left(\phi^{*} \nabla\right)_{j}\left(\mathcal{R}(\phi)\left(\partial_{m_{1}} \phi, \partial_{j} \phi\right)\left(\phi^{*} \nabla\right)_{\tilde{\mathbf{m}}}^{k-1} \phi\right)\right] .
\end{aligned}
$$

When $k=1$,

$$
\mathfrak{c}_{s, m_{1}}^{1}=0
$$

by (3.6). By the same we also get

$$
\mathfrak{c}_{m_{1}, j}^{1}=\mathcal{R}(\phi)\left(\partial_{m_{1}} \phi, \partial_{j} \phi\right) \partial_{j} \phi .
$$

In view of (3.14), (3.17), (3.18), and bounded geometry, we see that representation (3.7) and bound (3.8) hold when $k=1$. For $k>1$, (3.7) and (3.8) follow from induction. In particular, we apply (3.15) and (3.16) iteratively in (3.14), replacing $\partial_{s} \phi$ in (3.15) by $\left(\phi^{*} \nabla\right)_{j} \partial_{j} \phi$ courtesy of (1.1).

We obtain the following inequalities as a corollary. For convenience we introduce

$$
\mathbf{E}_{k}(s):=\int_{\mathbf{R}^{2}} \mathbf{e}_{k}(s, x) d x .
$$

Corollary 3.2. Let $\phi$ be a heat flow. Then for each $k \geq 1$ we have

$$
\begin{aligned}
\partial_{s} \mathbf{e}_{k}-\Delta \mathbf{e}_{k}+2 \mathbf{e}_{k+1} & \lesssim k \sum_{j=3}^{k+2} \sum_{a_{1}+\cdots+a_{j}=k+2}\left(\mathbf{e}_{a_{1}} \cdots \mathbf{e}_{a_{j}} \mathbf{e}_{k}\right)^{1 / 2} \\
\partial_{s} \mathbf{E}_{k}+2 \mathbf{E}_{k+1} & \lesssim k \sum_{j=3}^{k+2} \sum_{a_{1}+\cdots+a_{j}=k+2} \int_{\mathbf{R}^{2}}\left(\mathbf{e}_{a_{1}} \cdots \mathbf{e}_{a_{j}} \mathbf{e}_{k}\right)^{1 / 2} d x, \\
\mathbf{E}_{k}(s)-\mathbf{E}_{k}(r)+2 & \int_{r}^{s} \mathbf{E}_{k+1}\left(s^{\prime}\right) d s^{\prime} \\
& \lesssim k \sum_{j=3}^{k+2} \sum_{a_{1}+\cdots+a_{j}=k+2} \int_{r}^{s} \int_{\mathbf{R}^{2}}\left(\mathbf{e}_{a_{1}} \cdots \mathbf{e}_{a_{j}} \mathbf{e}_{k}\right)^{1 / 2} d x d s^{\prime},
\end{aligned}
$$

for $0 \leq r \leq s$.

Proof. Inequality (3.20) follows from (3.7) and (3.8). The remaining inequalities follow from integrating in (3.20).

The following corollary and its proof are essentially contained in [26, Corollary 3.6]. We present the statement and proof here for convenience and to introduce the diamagnetic inequality, which reappears in subsequent arguments. 
Corollary 3.3. Let $\phi$ be a heat flow. Then for each $k \geq 1$ we have

$$
\partial_{s} \sqrt{\mathbf{e}_{k}}-\Delta \sqrt{\mathbf{e}_{k}} \leq C_{k} \sum_{j=3}^{k+2} \sum_{a_{1}+\cdots+a_{j}=k+2}\left(\mathbf{e}_{a_{1}} \cdots \mathbf{e}_{a_{j}}\right)^{1 / 2}
$$

in the distributional sense for some constant $C_{k}>0$.

Proof. We shall only work formally; our arguments may be justified by replacing $\sqrt{\mathbf{e}_{k}}$ by $\sqrt{\epsilon^{2}+\mathbf{e}_{k}}$ and taking distributional limits sending $\epsilon \rightarrow 0$. Writing

$$
\partial_{s} \mathbf{e}_{k}=2 \sqrt{\mathbf{e}_{k}} \partial_{s} \sqrt{\mathbf{e}_{k}} \quad \text { and } \quad \Delta \mathbf{e}_{k}=2 \sqrt{\mathbf{e}_{k}} \Delta \sqrt{\mathbf{e}_{k}}+2\left|\partial_{x} \sqrt{\mathbf{e}_{k}}\right|^{2},
$$

we have

$$
\partial_{s} \sqrt{\mathbf{e}_{k}}-\Delta \sqrt{\mathbf{e}_{k}}+\frac{\mathbf{e}_{k+1}-\left|\partial_{x} \sqrt{\mathbf{e}_{k}}\right|^{2}}{\sqrt{\mathbf{e}_{k}}} \lesssim k \sum_{j=3}^{k+2} \sum_{a_{1}+\cdots+a_{j}=k+2}\left(\mathbf{e}_{a_{1}} \cdots \mathbf{e}_{a_{j}}\right)^{1 / 2} .
$$

The diamagnetic inequality

$$
\left|\partial_{x} \sqrt{\mathbf{e}_{k}}\right| \leq \sqrt{\mathbf{e}_{k+1}}
$$

follows from the Leibniz rule (3.5) and Cauchy-Schwarz:

$$
\left|\partial_{x} \mathbf{e}_{k}\right| \leq 2 \sqrt{\mathbf{e}_{k}} \sqrt{\mathbf{e}_{k+1}} .
$$

Using (3.25) in (3.24) implies (3.23).

Using positivity of the heat kernel (2.4) (or the maximum principle), we derive from (3.23) a Duhamel-type inequality (cf. (2.9))

$$
\sqrt{\mathbf{e}_{k}} \leq e^{\left(s_{1}-s_{0}\right) \Delta} \sqrt{\mathbf{e}_{k}}\left(s_{0}\right)+C_{k} \int_{s_{0}}^{s_{1}} e^{\left(s_{1}-s\right) \Delta} \sum_{j=3}^{k+2} \sum_{a_{1}+\cdots+a_{j}=k+2}\left(\mathbf{e}_{a_{1}} \cdots \mathbf{e}_{a_{j}}\right)^{1 / 2}(s) d s .
$$

\section{LOCAL THEORY AND CONTINUATION}

We begin with a standard local existence and uniqueness result.

Theorem 4.1. Let $\phi_{0}: \mathbf{R}^{2} \rightarrow \mathcal{M} \hookrightarrow_{\iota} \mathbf{R}^{n}$ differ from $\phi(\infty) \in \mathcal{M}$ by a Schwartz function. Then there exists an $S>0$ and a unique smooth heat flow extension $\phi:[0, S] \times \mathbf{R}^{2} \rightarrow \mathcal{M} \hookrightarrow{ }_{\iota} \mathbf{R}^{n}$ such that for each fixed $s \in S$ the function $\phi(s)-\phi(\infty)$ is Schwartz. Moreover, $\phi$ may be smoothly continued in time provided $\partial_{x} \phi$ remains bounded.

Proof. Rewrite (1.4) using the Duhamel formula (2.9) as

$$
\phi(s)=e^{s \Delta} \phi(0)+\int_{0}^{s} e^{\left(s-s^{\prime}\right) \Delta}\left(\Pi(\phi)\left(\partial_{x} \phi, \partial_{x} \phi\right)\right)\left(s^{\prime}\right) d s^{\prime} .
$$


As $\phi(0)=\phi_{0}$ differs from a Schwartz function by a constant, the linear solution $e^{s \Delta} \phi_{0}$ has all derivatives uniformly bounded. Using (2.7) and Picard iteration establishes local existence and uniqueness in the space $C_{s}^{0} C_{x}^{1}\left([0, S] \times \mathbf{R}^{2}\right)$ for sufficiently small $S$. In view of the fact that $\iota$ is a uniform isometric embedding, differentiating (4.1) and using the higher order parabolic regularity estimates (2.5) leads to the conclusion that $\phi$ is smooth and has all derivatives bounded on $[0, S]$. By working in weighted spaces, e.g. $\left\|\langle x\rangle^{k}\right\|_{C_{s}^{0} C_{x}^{\ell}}\left([0, S] \times \mathbf{R}^{2}\right)$, one can show that $\phi(s)-\phi(\infty)$ is rapidly decreasing in space for each fixed $s$, which, combined with boundedness of derivatives and Taylor's theorem with remainder ensures that $\phi(s)$ is Schwartz.

From the usual iteration argument and the compactness assumption it follows that $\phi$ may be continued in time and moreover remains Schwartz so long as $\partial_{x} \phi$ remains bounded.

4.1. Small energy results. The purpose of this subsection is to establish the following

Theorem 4.2. Let $\phi_{0}: \mathbf{R}^{2} \rightarrow \mathcal{M}$ be classical initial data. Then for $E_{0}:=$ $E\left(\phi_{0}\right)$ sufficiently small there exists a unique smooth heat flow extension $\phi: \mathbf{R}^{+} \times \mathbf{R}^{2} \rightarrow \mathcal{M}$ such that $\phi(s) \rightarrow_{s \rightarrow \infty} \phi(\infty)$ in the $C_{x}^{\infty}\left(\mathbf{R}^{2} \rightarrow \mathcal{M}\right)$ topology and such that for $k \geq 1$ the heat flow extension $\phi$ satisfies the following energy estimates:

$$
\begin{array}{r}
\int_{0}^{\infty} \int_{\mathbf{R}^{2}} s^{k-1} \mathbf{e}_{k+1}(s, x) d x d s \lesssim_{k} E_{0}, \\
\sup _{0<s<\infty} s^{k-1} \int_{\mathbf{R}^{2}} \mathbf{e}_{k}(s, x) d x \lesssim_{k} E_{0}, \\
\sup _{\substack{0<s<\infty \\
x \in \mathbf{R}^{2}}} s^{k} \mathbf{e}_{k}(s, x) \lesssim_{k} E_{0}, \\
\int_{0}^{\infty} s^{k-1} \sup _{x \in \mathbf{R}^{2}} \mathbf{e}_{k}(s, x) d s \lesssim_{k} E_{0} .
\end{array}
$$

Our first step towards proving the theorem is to establish some a priori estimates on energy densities under the assumption that the initial data has sufficiently small energy. The proofs resemble closely arguments found in [26, §4]. The present setting, however, demands several adaptations and also some additions, e.g. the proof of Proposition 4.8, which has no counterpart in [26]. After the a priori estimates are established, the global existence, uniqueness, and smoothness claims of the theorem follow easily from a continuity argument. The convergence claim and the family of estimates (4.5) will be taken up last and will follow respectively from (4.4) and (4.2). 
Let $I$ be a connected time interval containing the subinterval $[0, r]$, and let $\phi: I \times \mathbf{R}^{2} \rightarrow \mathcal{M}$ be a heat flow with initial energy $E_{0}$ at time $s=0$. Assume that on $[0, r]$ the covariant parabolic regularity estimates

$$
\begin{array}{r}
\int_{0}^{r} \int_{\mathbf{R}^{2}} s^{k-1} \mathbf{e}_{k+1}(s, x) d x d s \leq \sqrt{E_{0}}, \\
\sup _{0<s<r} s^{k-1} \int_{\mathbf{R}^{2}} \mathbf{e}_{k}(s, x) d x \leq \sqrt{E_{0}}, \\
\sup _{\substack{0<s<r \\
x \in \mathbf{R}^{2}}} s^{k} \mathbf{e}_{k}(s, x) \leq \sqrt{E_{0}}
\end{array}
$$

hold for $k=1,2, \ldots, N$, with, say, $N=10$. For each such $k$ we respectively label these statements $\mathbf{A}_{k}, \mathbf{B}_{k}$, and $\mathbf{C}_{k}$. Let $\widetilde{\mathbf{A}}_{k}, \widetilde{\mathbf{B}}_{k}$ and $\widetilde{\mathbf{C}}_{k}$ respectively refer to the similar but stronger (taking $E_{0}$ small) statements

$$
\begin{gathered}
\int_{0}^{r} \int_{\mathbf{R}^{2}} s^{k-1} \mathbf{e}_{k+1}(s, x) d x d s \lesssim_{k} E_{0}, \\
\sup _{0<s<r} s^{k-1} \int_{\mathbf{R}^{2}} \mathbf{e}_{k}(s, x) d x \lesssim_{k} E_{0}, \\
\sup _{\substack{0<s<r \\
x \in \mathbf{R}^{2}}} s^{k} \mathbf{e}_{k}(s, x) \lesssim_{k} E_{0},
\end{gathered}
$$

where the implied constants are only allowed to depend upon the integer $k$ and the topological and geometric properties of $\mathcal{M}$. Note that $\mathbf{A}_{k}, \mathbf{B}_{k}$, and $\mathbf{C}_{k}, k=1, \ldots, N$ may always be made to hold simultaneously by taking $r$ sufficiently small. We therefore assume now and throughout this subsection that this has been done. In addition to the above inequalities we shall need the integrated $L_{x}^{\infty}$ parabolic regularity estimate

$$
\int_{0}^{r}\left\|\mathbf{e}_{1}(s)\right\|_{L_{x}^{\infty}\left(\mathbf{R}^{2}\right)} d s \leq 1
$$

and so we choose $r$ even smaller if necessary so that this holds as well. We shall improve (4.9) to

$$
\int_{0}^{r}\left\|\mathbf{e}_{1}(s)\right\|_{L_{x}^{\infty}\left(\mathbf{R}^{2}\right)} d s \lesssim E_{0}
$$

From (3.21) and symmetry it follows that

$$
\partial_{s} \mathbf{E}_{k}+2 \mathbf{E}_{k+1} \lesssim k \sum_{j=3}^{k+2} \sum_{\substack{a_{1}+\cdots+a_{j}=k+2 \\ a_{1} \leq \cdots \leq a_{j}}} \int_{\mathbf{R}^{2}}\left(\mathbf{e}_{a_{1}} \cdots \mathbf{e}_{a_{j}} \mathbf{e}_{k}\right)^{1 / 2} d x .
$$

We show that together $\mathbf{A}_{k}, \mathbf{B}_{k}, \mathbf{C}_{k}, k=1, \ldots N$, and (4.9) imply the a priori estimates $\widetilde{\mathbf{A}}_{k}, \widetilde{\mathbf{B}}_{k}, \widetilde{\mathbf{C}}_{k}, k=1, \ldots, N$, and (4.10). This we prove through a sequence of propositions: Proposition 4.5 establishes that $\widetilde{\mathbf{B}}_{1}$ is true. In Propositions 4.7 and 4.8 respectively, $\widetilde{\mathbf{B}}_{1}$ and (4.9) are used to show that $\mathbf{A}_{1}$ and (4.10) hold. By using $\widetilde{\mathbf{A}}_{1}$, Proposition 4.3 concludes that $\widetilde{\mathbf{A}}_{k}, k=2, \ldots, N$ 
hold. The $\widetilde{\mathbf{A}}_{k}$ 's then imply $\widetilde{\mathbf{B}}_{j}, j=1, \ldots, N$ according to Proposition 4.4. Finally, the $\widetilde{\mathbf{B}}_{k}$ 's are used to establish $\widetilde{\mathbf{C}}_{j}, j=1, \ldots, N$ in Proposition 4.6 .

Assume now that $k>1$ so that $a_{j}>1$. Using the hypotheses $\mathbf{C}_{j}$ for $j<k$, we conclude

$$
2 \mathbf{E}_{k+1}+\partial_{s} \mathbf{E}_{k} \lesssim k \sqrt{E_{0}} \sum_{a=2}^{k} \int_{\mathbf{R}^{2}} s^{-(k+2-a) / 2} \mathbf{e}_{a}^{1 / 2} \mathbf{e}_{k}^{1 / 2} d x .
$$

Applying Cauchy-Schwarz yields

$$
2 \mathbf{E}_{k+1}+\partial_{s} \mathbf{E}_{k} \lesssim k \sqrt{E_{0}} \sum_{a=2}^{k} s^{-(k+2-a) / 2} \mathbf{E}_{a}^{1 / 2} \mathbf{E}_{k}^{1 / 2},
$$

and so by the arithmetic- geometric-mean inequality,

$$
2 \mathbf{E}_{k+1}+\partial_{s} \mathbf{E}_{k} \lesssim k \sqrt{E_{0}} \sum_{a=2}^{k} s^{a-k-1} \mathbf{E}_{a} .
$$

Inequality (4.11) will be used extensively in the proofs below.

Proposition 4.3. Suppose that $\widetilde{\mathbf{A}}_{1}$ holds. Then $\widetilde{\mathbf{A}}_{k}$ holds for $k=2,3, \ldots, N$.

Proof. Let $\rho$ be a smooth nonnegative cutoff function supported on $[1 / 2,2]$ and bounded by 1 and let $s_{0} \in(0, r / 2)$. From (4.11) it follows that

$$
\begin{aligned}
s_{0}^{k-1} \int_{0}^{r} \rho\left(\frac{s}{s_{0}}\right) \mathbf{E}_{k+1} d s- & s_{0}^{k-2} \int_{0}^{r}\left(\partial_{s} \rho\right)\left(\frac{s}{s_{0}}\right) \mathbf{E}_{k}(s) d s \\
& \lesssim_{k} \sqrt{E_{0}} \sum_{a=2}^{k} s_{0}^{a-2} \int_{0}^{r} \rho\left(\frac{s}{s_{0}}\right) \mathbf{E}_{a}(s) d s
\end{aligned}
$$

which by the triangle inequality may be rewritten as

$$
\begin{aligned}
s_{0}^{k-1} \int_{0}^{r} \rho\left(\frac{s}{s_{0}}\right) \mathbf{E}_{k+1} d s \lesssim & \sqrt{E_{0}} \sum_{a=2}^{k} s_{0}^{a-2} \int_{0}^{r} \rho\left(\frac{s}{s_{0}}\right) \mathbf{E}_{a}(s) d s \\
& +\left|s_{0}^{k-2} \int_{0}^{r}\left(\partial_{s} \rho\right)\left(\frac{s}{s_{0}}\right) \mathbf{E}_{k}(s) d s\right| .
\end{aligned}
$$

Using induction, apply $\mathbf{A}_{1}, \mathbf{A}_{2}, \ldots, \mathbf{A}_{k-2}$ to respectively bound the integrals of $\mathbf{E}_{2}, \mathbf{E}_{3}, \ldots, \mathbf{E}_{k-1}$ in the right hand side hand side and use $\widetilde{\mathbf{A}}_{k-1}$ to bound the integral of $\mathbf{E}_{k}$. Summing dyadically in $s_{0}$ completes the proof.

Proposition 4.4. Let $k>1$ and assume that $\widetilde{\mathbf{A}}_{k-1}$ and $\widetilde{\mathbf{A}}_{k}$ hold. Then $\widetilde{\mathbf{B}}_{k}$ holds. 
Proof. From (4.11) it follows that for any $s_{0}$ in the range $0<s_{0}<r$ we have

$2 \int_{0}^{s_{0}} s^{k-1} \mathbf{E}_{k+1} d s-\int_{0}^{s_{0}}(k-1) s^{k-2} \mathbf{E}_{k} d s+s_{0}^{k-1} \mathbf{E}_{k}\left(s_{0}\right) \lesssim k \sqrt{E_{0}} \sum_{a=2}^{k} \int_{0}^{s_{0}} s^{a-2} \mathbf{E}_{a} d s$.

Applying hypotheses $\mathbf{A}_{1}, \mathbf{A}_{2}, \ldots, \mathbf{A}_{k-1}$ to control the integrals on the right hand side and $\widetilde{\mathbf{A}}_{k-1}$ and $\widetilde{\mathbf{A}}_{k}$ to control those on the left implies by the triangle inequality that

$$
s_{0}^{k-1} \mathbf{E}_{k}\left(s_{0}\right) \lesssim_{k} E_{0}
$$

thereby establishing $\widetilde{\mathbf{B}}_{k}$.

Proposition 4.5. Inequality $\widetilde{\mathbf{B}}_{1}$ holds.

Proof. This follows from the well-known property of energy monotonicity (see for instance [20]), established using the observation that the heat flow equation is the $L^{2}$-gradient flow associated to the energy functional (1.6):

$$
\begin{aligned}
\int_{\mathbf{R}^{2}}\left\langle\partial_{s} \phi, \partial_{s} \phi\right\rangle_{\phi^{*} h} d x & =\int_{\mathbf{R}^{2}}\left\langle\left(\phi^{*} \nabla\right)_{j} \partial_{j} \phi, \partial_{s} \phi\right\rangle_{\phi^{*} h} d x \\
& =-\int_{\mathbf{R}^{2}}\left\langle\partial_{j} \phi,\left(\phi^{*} \nabla\right)_{j} \partial_{s} \phi\right\rangle_{\phi^{*} h} d x \\
& =-\int_{\mathbf{R}^{2}}\left\langle\partial_{j} \phi,\left(\phi^{*} \nabla\right)_{s} \partial_{j} \phi\right\rangle_{\phi^{*} h} d x \\
& =-\frac{1}{2} \partial_{s} \int_{\mathbf{R}^{2}} \mathbf{e}_{1} d x .
\end{aligned}
$$

Proposition 4.6. Inequality $\widetilde{\mathbf{B}}_{1}$ implies $\widetilde{\mathbf{C}}_{1}$. For $k>1$ the inequalities $\widetilde{\mathbf{B}}_{1}, \widetilde{\mathbf{B}}_{2}, \ldots, \widetilde{\mathbf{B}}_{k}$ and $\widetilde{\mathbf{C}}_{1}, \widetilde{\mathbf{C}}_{2}, \ldots, \widetilde{\mathbf{C}}_{k-1}$ together imply $\widetilde{\mathbf{C}}_{k}$.

Proof. Applying Duhamel (3.26) and parabolic regularity (2.5) to (3.23), we get

$$
\begin{aligned}
\left\|\sqrt{\mathbf{e}_{k}}\left(s_{0}\right)\right\|_{L_{x}^{\infty}} \lesssim_{k} & s_{0}^{-1 / 2}\left\|\sqrt{\mathbf{e}_{k}}\left(s_{0} / 2\right)\right\|_{L_{x}^{2}} \\
& +\int_{s_{0} / 2}^{s_{0}} \sum_{j=3}^{k+2} \sum_{a_{1}+\cdots+a_{j}=k+2}\left(s_{0}-s\right)^{-1 / 2}\left\|\left(\mathbf{e}_{a_{1}} \cdots \mathbf{e}_{a_{j}}\right)^{1 / 2}\right\|_{L_{x}^{2}} d s .
\end{aligned}
$$

To bound the first term on the right hand side, use $\widetilde{\mathbf{B}}_{k}$. For the remaining terms, use the hypotheses $\widetilde{\mathbf{B}}_{j}, \widetilde{\mathbf{C}}_{j}$.

Proposition 4.7. Inequalities $\widetilde{\mathbf{B}}_{1}$ and 4.9 imply $\widetilde{\mathbf{A}}_{1}$. 
Proof. Here we apply (3.22) with $k=1$. By $\widetilde{\mathbf{B}}_{1}$ and the triangle inequality, we need only control the right hand side by $E_{0}$ :

$$
\begin{aligned}
\int_{0}^{r} \int_{\mathbf{R}^{2}} \mathbf{e}_{1}^{2}(s, x) d x d s & \leq \int_{0}^{r} \sup _{x} \mathbf{e}_{1}(s, x) \int_{\mathbf{R}^{2}} \mathbf{e}_{1}(s, x) d x d s \\
& \leq 2 E(s) \int_{0}^{r} \sup _{x} \mathbf{e}_{1}(s, x) d s \\
& \lesssim E_{0},
\end{aligned}
$$

where the last inequality follows from $\widetilde{\mathbf{B}}_{1}$ and (4.9).

Proposition 4.8. Inequalities $\widetilde{\mathbf{B}}_{1}$ and (4.9) imply (4.10).

Proof. In view of (3.23) with $k=1$ we apply Duhamel (3.26) to $\sqrt{\mathbf{e}_{1}}$ and obtain

$$
\sqrt{\mathbf{e}_{1}}(s, x) \leq e^{s \Delta} \sqrt{\mathbf{e}_{1}}(0, x)+C_{1} \int_{0}^{s} e^{\left(s-s^{\prime}\right) \Delta}{\sqrt{\mathbf{e}_{1}}}^{3}\left(s^{\prime}, x\right) d s^{\prime} .
$$

Taking $L_{x}^{\infty}$ norms and squaring gives

$$
\left\|\mathbf{e}_{1}(s)\right\|_{L_{x}^{\infty}} \lesssim\left\|e^{s \Delta} \sqrt{\mathbf{e}_{1}}(0)\right\|_{L_{x}^{\infty}}^{2}+\left\|\int_{0}^{s} e^{\left(s-s^{\prime}\right) \Delta}{\sqrt{\mathbf{e}_{1}}}^{3}\left(s^{\prime}, x\right) d s^{\prime}\right\|_{L_{x}^{\infty}}^{2} .
$$

We now integrate in $s$ and bound each of the terms of the right hand side separately. Using the $p=\infty$ case of (2.8) yields

$$
\int_{0}^{r}\left\|e^{s \Delta} \sqrt{\mathbf{e}_{1}}(0)\right\|_{L_{x}^{\infty}}^{2} d s \lesssim\left\|\sqrt{\mathbf{e}_{1}}(0)\right\|_{L_{x}^{2}}^{2}=2 E_{0}
$$

independently of $r$. By two applications of Minkowski's inequality, we have for the remaining term that

$$
\begin{aligned}
I_{2} & :=\int_{0}^{r}\left\|\int_{0}^{s} e^{\left(s-s^{\prime}\right) \Delta}{\sqrt{\mathbf{e}_{1}}}^{3}\left(s^{\prime}, x\right) d s^{\prime}\right\|_{L_{x}^{\infty}}^{2} d s \\
& \leq \int_{0}^{r}\left(\int_{0}^{r}\left\|e^{\left(s-s^{\prime}\right) \Delta}{\sqrt{\mathbf{e}_{1}}}^{3}\left(s^{\prime}, x\right)\right\|_{L_{x}^{\infty}} d s^{\prime}\right)^{2} d s \\
& \leq\left(\int_{0}^{r}\left(\int_{0}^{r}\left\|e^{\left(s-s^{\prime}\right) \Delta}{\sqrt{\mathbf{e}_{1}}}^{3}\left(s^{\prime}, x\right)\right\|_{L_{x}^{\infty}}^{2} d s\right)^{\frac{1}{2}} d s^{\prime}\right)^{2}
\end{aligned}
$$

By (2.8) with $p=\infty$, it follows that

$$
\int_{0}^{r}\left\|e^{\left(s-s^{\prime}\right) \Delta}{\sqrt{\mathbf{e}_{1}}}^{3}\left(s^{\prime}, x\right)\right\|_{L_{x}^{\infty}}^{2} d s \lesssim\left\|{\sqrt{\mathbf{e}_{1}}}^{3}\left(s^{\prime}\right)\right\|_{L_{x}^{2}}^{2} .
$$


Thus

$$
\begin{aligned}
I_{2} & \lesssim\left(\int_{0}^{r}\left\|{\sqrt{\mathbf{e}_{1}}}^{3}\left(s^{\prime}\right)\right\|_{L_{x}^{2}} d s^{\prime}\right)^{2} \\
& \leq\left(\int_{0}^{r}\left\|\sqrt{\mathbf{e}_{1}}\left(s^{\prime}\right)\right\|_{L_{x}^{\infty}}^{2}\left\|\sqrt{\mathbf{e}_{1}}\left(s^{\prime}\right)\right\|_{L_{x}^{2}} d s^{\prime}\right)^{2} \\
& \leq \sup _{s}\left\|\sqrt{\mathbf{e}_{1}}(s)\right\|_{L_{x}^{2}}^{2} \cdot\left(\int_{0}^{r}\left\|\mathbf{e}_{1}\left(s^{\prime}\right)\right\|_{L_{x}^{\infty}} d s^{\prime}\right)^{2} \\
& \leq 2 E_{0}\left(\int_{0}^{r}\left\|\mathbf{e}_{1}\left(s^{\prime}\right)\right\|_{L_{x}^{\infty}} d s^{\prime}\right)^{2},
\end{aligned}
$$

where the last inequality holds because of $\widetilde{\mathbf{B}}_{1}$. Therefore

$$
\int_{0}^{r}\left\|\mathbf{e}_{1}(s)\right\|_{L_{x}^{\infty}} d s \lesssim E_{0}+E_{0}\left(\int_{0}^{r}\left\|\mathbf{e}_{1}(s)\right\|_{L_{x}^{\infty}} d s\right)^{2},
$$

and so in view of (4.9) it follows that for $E_{0}$ sufficiently small

$$
\int_{0}^{r}\left\|\mathbf{e}_{1}(s)\right\|_{L_{x}^{\infty}} d s \lesssim E_{0}
$$

independently of $r$.

Proof. (Proof of Theorem 4.2). Via a straightforward continuity argument, Theorem 4.1 together with Propositions 4.34 .8 imply the global existence and uniqueness claims of Theorem 4.2 along with (4.2)-(4.4) for $k=1,2, \ldots, N$ and (4.5) for $k=1$. As $N$ may be taken arbitrarily large, inequalities (4.2)(4.4) in fact hold for all $k \geq 1$. Convergence to $\phi(\infty)$ as $s \rightarrow \infty$ in the $C_{x}^{\infty}\left(\mathbf{R}^{2} \rightarrow \mathcal{M}\right)$ topology is an easy consequence of the energy estimates (4.4).

It remains to establish (4.5) for $k>1$. These follow as a corollary from (4.2) and are shown in the following proposition.

Proposition 4.9. Let $\phi$ be a heat flow with classical initial data. Then for sufficiently small initial energy $E_{0}$, we have

$$
\int_{0}^{\infty} s^{k-1}\left\|\mathbf{e}_{k}(s)\right\|_{L_{x}^{\infty}\left(\mathbf{R}^{2}\right)} d s \lesssim_{k} E_{0}
$$

for $k \geq 1$.

Proof. We have already established the $k=1$ case. For $k>1$, the proof procedes as in [26] and for convenience we reproduce the short argument here.

The Gagliardo-Nirenberg inequality (2.2) implies

$$
\left\|\sqrt{\mathbf{e}_{k}}(s)\right\|_{L_{x}^{\infty}} \lesssim\left\|\sqrt{\mathbf{e}_{k}}(s)\right\|_{L_{x}^{2}}^{1 / 3}\left\|\partial_{x} \sqrt{\mathbf{e}_{k}}(s)\right\|_{L_{x}^{4}}^{2 / 3}
$$


and so in view of the diamagnetic inequality (3.25) it holds that

$$
\left\|\sqrt{\mathbf{e}_{k}}(s)\right\|_{L_{x}^{\infty}} \lesssim\left\|\sqrt{\mathbf{e}_{k}}(s)\right\|_{L_{x}^{2}}^{1 / 3}\left\|\sqrt{\mathbf{e}_{k+1}}(s)\right\|_{L_{x}^{4}}^{2 / 3} .
$$

From the Gagliardo-Nirenberg inequality (2.3) we have

$$
\left\|\sqrt{\mathbf{e}_{k+1}}(s)\right\|_{L_{x}^{4}} \lesssim\left\|\sqrt{\mathbf{e}_{k+1}}(s)\right\|_{L_{x}^{2}}^{1 / 2}\left\|\partial_{x} \sqrt{\mathbf{e}_{k+1}}(s)\right\|_{L_{x}^{2}}^{1 / 2}
$$

Combining the above inequalities yields

$$
\left\|\sqrt{\mathbf{e}_{k}}(s)\right\|_{L_{x}^{\infty}} \lesssim\left\|\sqrt{\mathbf{e}_{k}}(s)\right\|_{L_{x}^{2}}^{1 / 3}\left\|\sqrt{\mathbf{e}_{k+1}}(s)\right\|_{L_{x}^{2}}^{1 / 3}\left\|\sqrt{\mathbf{e}_{k+2}}(s)\right\|_{L_{x}^{2}}^{1 / 3} .
$$

Using (4.14) in (4.13), we conclude with Hölder's inequality and (4.2) and (4.3).

4.2. A controlling quantity. Define over any time interval $I$ the following "norm":

$$
\|\phi\|_{\mathcal{E}(I)}:=\left\|\left|\partial_{x} \phi\right|_{\phi^{*} h}\right\|_{L_{s, x}^{4}\left(I \times \mathbf{R}^{2}\right)} .
$$

In terms of energy densities, we may equivalently write

$$
\begin{aligned}
\|\phi\|_{\mathcal{E}(I)} & =\left\|\sqrt{\mathbf{e}_{1}}\right\|_{L_{s, x}^{4}\left(I \times \mathbf{R}^{2}\right)} \\
& =\left\|\mathbf{e}_{1}\right\|_{L_{s, x}^{2}\left(I \times \mathbf{R}^{2}\right)}^{\frac{1}{2}} .
\end{aligned}
$$

Strictly speaking, $\mathcal{E}$ is not a norm or even a seminorm on smooth maps $I \times \mathbf{R}^{2} \rightarrow \mathcal{M}$, but can be interpreted as an intrinsic nonlinear analogue of the $\dot{W}_{x}^{1,4}$ seminorm. Abusing terminology, we refer to $\mathcal{E}$ as the $\mathcal{E}$-norm. Note that the $\mathcal{E}$-norm is critical, as it is invariant with respect to the scaling (1.3). It is also clear that the $\mathcal{E}$-norm is monotonic in the sense that it is non-decreasing in time.

In the following let $\phi$ be a partial heat flow whose initial data $\phi(0)=\phi_{0}$ has initial energy $E_{0}:=E\left(\phi_{0}\right)$. In this subsection we shall show that the various energy quantities comprising the left-hand-sides of (4.2)-(4.5) are all controlled by the $\mathcal{E}$-norm of $\phi$. Throughout we use

$$
\mathcal{E}_{[0, r)}:=\|\phi\|_{\mathcal{E}([0, r))}
$$

as a notational shorthand. Note that in this subsection we do not require $E_{0}<E_{\text {crit }}$ : the results are valid so long as the $\mathcal{E}$-norm is finite. However, in applications in this paper we shall take $E_{0}<E_{\text {crit }}$ and in 6 we prove finiteness of the $\mathcal{E}$-norm under this assumption. For simplicity the following propositions are stated assuming the initial data is given at initial time $s=0$, though the proofs are valid for any initial time $s=s_{0} \geq 0$ we might choose.

Proposition 4.10. Let $\phi$ be a heat flow on $[0, r)$ with initial energy $E_{0}$. Then

$$
\int_{0}^{r} \mathbf{E}_{2}(s) d s \lesssim E_{0}, \mathcal{E}_{[0, r)} 1 .
$$


Proof. Using (3.22) with $k=1$, we have

$$
2 \int_{0}^{r} \mathbf{E}_{2}(s) d s+\mathbf{E}_{1}(r)-2 E_{0} \lesssim\|\phi\|_{\mathcal{E}([0, r))}^{4} .
$$

By monotonicity of the energy $\mathbf{E}_{1} / 2$ the claim follows.

Proposition 4.11. Let $\phi$ be a heat flow on $[0, r)$ with initial energy $E_{0}$. Then

$$
\left\|\mathbf{e}_{1}\right\|_{L_{s}^{1} L_{x}^{\infty}\left([0, r) \times \mathbf{R}^{2}\right)} \lesssim E_{0}, \mathcal{E}_{[0, r)} 1
$$

Proof. Due to Duhamel (3.26) we have for all $s>r_{0} \geq 0$ that

$$
\left\|\mathbf{e}_{1}(s)\right\|_{L_{x}^{\infty}} \lesssim\left\|e^{s \Delta} \sqrt{\mathbf{e}_{1}}\left(r_{0}\right)\right\|_{L_{x}^{\infty}}^{2}+\left\|\int_{r_{0}}^{s} e^{\left(s-s^{\prime}\right) \Delta}{\sqrt{\mathbf{e}_{1}^{3}}}^{3}\left(s^{\prime}, x\right) d s^{\prime}\right\|_{L_{x}^{\infty}}^{2} .
$$

We upper-bound the nonlinear term using

$$
\left\|\int_{r_{0}}^{s} e^{\left(s-s^{\prime}\right) \Delta}{\sqrt{\mathbf{e}_{1}}}^{3}\left(s^{\prime}, x\right) d s^{\prime}\right\|_{L_{x}^{\infty}}^{2} \leq\left(\int_{r_{0}}^{s}\left\|e^{\left(s-s^{\prime}\right) \Delta}{\sqrt{\mathbf{e}_{1}}}^{3}\left(s^{\prime}, x\right)\right\|_{L_{x}^{\infty}} d s^{\prime}\right)^{2} .
$$

Integrating in $s$ from $r_{0} \geq 0$ to $t \leq r$, we have by Minkowski's inequality that

$$
\begin{aligned}
& \int_{r_{0}}^{t}\left(\int_{r_{0}}^{s}\left\|e^{\left(s-s^{\prime}\right) \Delta}{\sqrt{\mathbf{e}_{1}}}^{3}\left(s^{\prime}, x\right)\right\|_{L_{x}^{\infty}} d s^{\prime}\right)^{2} d s \\
& \quad \leq\left(\int_{r_{0}}^{t}\left(\int_{s^{\prime}}^{t}\left\|e^{\left(s-s^{\prime}\right) \Delta}{\sqrt{\mathbf{e}_{1}}}^{3}\left(s^{\prime}, x\right)\right\|_{L_{x}^{\infty}}^{2} d s\right)^{1 / 2} d s^{\prime}\right)^{2} .
\end{aligned}
$$

From the (2.8) with $p=\infty$ it follows that

$$
\int_{s^{\prime}}^{t}\left\|e^{\left(s-s^{\prime}\right) \Delta}{\sqrt{\mathbf{e}_{1}}}^{3}\left(s^{\prime}, x\right)\right\|_{L_{x}^{\infty}}^{2} d s \lesssim\left\|{\sqrt{\mathbf{e}_{1}}}^{3}\left(s^{\prime}\right)\right\|_{L_{x}^{2}}^{2},
$$

and so the right hand side of (4.16) is controlled by

$$
\begin{aligned}
\left(\int_{r_{0}}^{t}\left\|{\sqrt{\mathbf{e}_{1}}}^{3}(s)\right\|_{L_{x}^{2}} d s\right)^{2} & \lesssim\left(\int_{r_{0}}^{t}\left\|\sqrt{\mathbf{e}_{1}}(s)\right\|_{L_{x}^{\infty}}\left\|\mathbf{e}_{1}(s)\right\|_{L_{x}^{2}} d s\right)^{2} \\
& \lesssim\left(\left(\int_{r_{0}}^{t}\left\|\sqrt{\mathbf{e}_{1}}(s)\right\|_{L_{x}^{\infty}}^{2} d s\right)^{1 / 2}\left(\int_{r_{0}}^{t}\left\|\mathbf{e}_{1}(s)\right\|_{L_{x}^{2}}^{2} d s\right)^{1 / 2}\right)^{2} \\
& =\int_{r_{0}}^{t}\left\|\mathbf{e}_{1}(s)\right\|_{L_{x}^{\infty}} d s \cdot \int_{r_{0}}^{t}\left\|\mathbf{e}_{1}(s)\right\|_{L_{x}^{2}}^{2} d s .
\end{aligned}
$$

By using (2.8) with $p=\infty$ to bound the linear term appearing in (4.15), we conclude

$$
\int_{r_{0}}^{t}\left\|\mathbf{e}_{1}(s)\right\|_{L_{x}^{\infty}} d s \lesssim E_{0}+\int_{r_{0}}^{t}\left\|\mathbf{e}_{1}(s)\right\|_{L_{x}^{\infty}} d s \cdot \int_{r_{0}}^{t}\left\|\mathbf{e}_{1}(s)\right\|_{L_{x}^{2}}^{2} d s .
$$


The proposition now follows from splitting up the time interval $[0, r)$ into $O\left(\left(\mathcal{E}_{[0, r)}\right)^{2}\right)$ intervals $I_{j}$ on which $\left\|\mathbf{e}_{1}\right\|_{L_{s}^{2} L_{x}^{2}\left(I_{j} \times \mathbf{R}^{2}\right)}$ is sufficiently small.

Proposition 4.12. Let $\phi$ be a heat flow on $[0, r)$ with initial energy $E_{0}$. Then

$$
\sup _{\substack{0<s<r \\ x \in \mathbf{R}^{2}}} s \mathbf{e}_{1}(s, x) \lesssim E_{0} \exp \left(2\left\|\mathbf{e}_{1}\right\|_{L_{s}^{1} L_{x}^{\infty}\left([0, r) \times \mathbf{R}^{2}\right)}\right) .
$$

Proof. Duhamel (3.26) and parabolic regularity (2.5) imply

$$
\left\|\sqrt{\mathbf{e}_{1}}(r)\right\|_{L_{x}^{\infty}} \lesssim r^{-1 / 2} E_{0}^{1 / 2}+\int_{0}^{r}\left\|e^{(r-s) \Delta}{\sqrt{\mathbf{e}_{1}}}^{3}(s)\right\|_{L_{x}^{\infty}} d s .
$$

By Young's inequality,

$$
\left\|\sqrt{\mathbf{e}_{1}}(r)\right\|_{L_{x}^{\infty}} \lesssim r^{-1 / 2} E_{0}^{1 / 2}+\int_{0}^{r}\left\|\sqrt{\mathbf{e}_{1}}(s)\right\|_{L_{x}^{\infty}} \cdot\left\|\mathbf{e}_{1}(s)\right\|_{L_{x}^{\infty}} d s,
$$

and so by Gronwall's inequality,

$$
\left\|\sqrt{\mathbf{e}_{1}}(r)\right\|_{L_{x}^{\infty}} \lesssim r^{-1 / 2} E_{0}^{1 / 2} \exp \left(\int_{0}^{r}\left\|\mathbf{e}_{1}(s)\right\|_{L_{x}^{\infty}} d s\right) .
$$

Lemma 4.13. Let $\phi: I \times \mathbf{R}^{2} \rightarrow \mathcal{M}$ be a heat flow with classical initial data $\phi_{0}$ with energy $E_{0}:=E\left(\phi_{0}\right)$ and suppose that $\|\phi\|_{\mathcal{E}(I)}<\infty$. Setting $S_{I}:=\|\phi\|_{\mathcal{E}(I)}$ for short, we have for each $k \geq 1$ that

$$
\begin{array}{r}
\int_{I} \int_{\mathbf{R}^{2}} s^{k-1} \mathbf{e}_{k+1}(s, x) d x d s \\
\sup _{s \in I} s^{k-1} \int_{\mathbf{R}^{2}} \mathbf{e}_{k}(s, x) d x, \lesssim_{E_{0}, k, S_{I}} 1, \\
\sup _{s \in I} s^{k} \mathbf{e}_{k}(s, x) \lesssim_{E_{0}, k, S_{I}} 1, \\
\int_{I \in \mathbf{R}^{2}} s^{k-1} \sup _{x \in \mathbf{R}^{2}} \mathbf{e}_{k}(s, x) d s \lesssim_{E_{0}, k, S_{I}} 1 .
\end{array}
$$

Proof. The proof is by induction. The base case $(k=1)$ of (4.17) follows from Proposition 4.10, that of (4.18) from Proposition 4.5, that of (4.19) from the argument of Proposition 4.6, and finally that of (4.20) from Proposition 4.11.

Now assume $k>1$ and that the inequalities of the lemma have been proven for all smaller $k$. Then we have at our disposal (4.11) as in \$4. Repeating the argument of Proposition 4.3 implies (4.17). The proof of (4.18) follows from the argument used in Proposition 4.4. Inequalities (4.19) follow from Proposition 4.6. Finally, we conclude (4.20) using the argument of Proposition 4.9. 


\section{LOCAL ENERGy ESTIMATES}

To prepare for the minimal blow-up solution argument of the next section we now establish some local energy estimates. In view of this and for the sake of convenience we introduce the new notation

$$
E(\phi(s) 1 U):=\frac{1}{2} \int_{U} \mathbf{e}_{1}(s) d x
$$

and similarly,

$$
\mathbf{E}_{k}(\phi(s) 1 U):=\int_{U} \mathbf{e}_{k}(s) d x
$$

where $U \subset \mathbf{R}^{2}$. Usually we take $U=B(x, R)$, that is, equal to an open ball in $\mathbf{R}^{2}$ centered at the point $x$ and of radius $R$. The following two lemmas are intrinsic formulations of [20, Lemmas 3.1, 3.2].

Lemma 5.1. (Energy localization). Let $\phi$ be a heat flow on $[0, S] \times \mathbf{R}^{2}$. Then for any $s \in[0, S]$ and $R>0$ we have

$$
\begin{aligned}
\left\|\mathbf{e}_{1}\right\|_{L_{s, x}^{2}\left([0, s] \times \mathbf{R}^{2}\right)}^{2} \lesssim & \left\|\mathbf{E}_{1}(\phi 1 B(\cdot, R))\right\|_{L_{s, x}^{\infty}\left([0, s] \times \mathbf{R}^{2}\right)} \times \\
& \times\left(\left\|\mathbf{e}_{2}\right\|_{L_{s, x}^{1}\left([0, s] \times \mathbf{R}^{2}\right)}+\frac{1}{R^{2}}\left\|\mathbf{e}_{1}\right\|_{L_{s, x}^{1}\left([0, s] \times \mathbf{R}^{2}\right)}\right) .
\end{aligned}
$$

Lemma 5.1 follows as a corollary from the following result via a simple covering argument as in [20].

Lemma 5.2. Let $\phi$ be a heat flow on $[0, S] \times \mathbf{R}^{2}$. Let $x \in \mathbf{R}^{2}, R>0$, set $B:=B(x, R)$, and let $\chi_{B}$ be the indicator function of $B$. Then for any $s \in[0, S]$ and $R>0$ we have

$$
\begin{aligned}
\left\|\mathbf{e}_{1} \cdot \chi_{B}\right\|_{L_{s, x}^{2}\left([0, s] \times \mathbf{R}^{2}\right)}^{2} \lesssim & \left\|\mathbf{E}_{1}(\phi 1 B(x, R))\right\|_{L_{s}^{\infty}([0, s])} \times \\
& \times\left(\left\|\mathbf{e}_{2} \cdot \chi_{B}\right\|_{L_{s, x}^{1}\left([0, s] \times \mathbf{R}^{2}\right)}+\frac{1}{R^{2}}\left\|\mathbf{e}_{1} \cdot \chi_{B}\right\|_{L_{s, x}^{1}\left([0, s] \times \mathbf{R}^{2}\right)}\right) .
\end{aligned}
$$

Proof. Letting

$$
\left(\sqrt{\mathbf{e}_{1}}\right)_{\text {ave }}:=\frac{1}{\pi R^{2}} \int_{B} \sqrt{\mathbf{e}_{1}} d x,
$$

we have by Poincaré's inequality that

$$
\begin{aligned}
\int_{0}^{s} \int_{B} \mathbf{e}_{1}^{2} d x d s^{\prime} \lesssim & \int_{0}^{s} \int_{B}\left|\sqrt{\mathbf{e}_{1}}-\left(\sqrt{\mathbf{e}_{1}}\right)_{\mathrm{ave}}\right|^{4} d x d s^{\prime}+\int_{0}^{s} \int_{B}\left(\sqrt{\mathbf{e}_{1}}\right)_{\text {ave }}^{4} d x d s^{\prime} \\
\lesssim & \sup _{0<s^{\prime}<s} \int_{B}\left|\sqrt{\mathbf{e}_{1}}-\left(\sqrt{\mathbf{e}_{1}}\right)_{\mathrm{ave}}\right|^{2} d x \int_{0}^{s} \int_{B}\left|\partial_{x} \sqrt{\mathbf{e}_{1}}\right|^{2} d x d s^{\prime} \\
& +\int_{0}^{s}\left(\pi R^{2}\right)^{-3}\left|\int_{B} \sqrt{\mathbf{e}_{1}} d x\right|^{4} d s^{\prime}
\end{aligned}
$$


Now

$$
\int_{B} \mid \sqrt{\mathbf{e}_{1}}-\left(\sqrt{\mathbf{e}_{1}}\right) \text { ave }\left.\right|^{2} d x \leq \int_{B} \mathbf{e}_{1} d x .
$$

From Hölder's inequality it follows that

$$
\begin{aligned}
\left|\int_{B} \sqrt{\mathbf{e}_{1}}\left(s^{\prime}\right) d x\right|^{4} & \leq\left(\pi R^{2}\right)^{2}\left(\int_{B} \mathbf{e}_{1} d x\right)^{2} \\
& \leq\left(\pi R^{2}\right)^{2}\left\|\int_{B} \mathbf{e}_{1}(\cdot) d x\right\|_{L_{s}^{\infty}\left(\left[0, s^{\prime}\right]\right)} \int_{B} \mathbf{e}_{1}\left(s^{\prime}\right) d x .
\end{aligned}
$$

Substituting (5.3) and (5.4) in (5.2) and using the diamagnetic inequality (3.25) in (5.2) proves the lemma.

Lemma 5.3. (Energy concentration). There exists a constant $C=C\left(\mathbf{R}^{2}, \mathcal{M}\right)$ such that for any $S>0$ and any heat flow $\phi$ on $[0, S] \times \mathbf{R}^{2}$ we have for any $0 \leq s \leq S, x \in \mathbf{R}^{2}$, and $R>0$ that

$$
E(\phi(s) 1 B(x, R)) \leq E(\phi(0) 1 B(x, 2 R))+C \frac{s}{R^{2}} E_{0}
$$

Proof. Let $\rho \in C_{c}^{\infty}\left(\mathbf{R}^{2} \rightarrow[0,1]\right)$ be compactly supported in $B_{2 R}(x)$, equal to 1 on $B_{R}(x)$, and such that $\left|\partial_{x} \rho\right| \leq 2 / R$. Then via (1.1), integration by parts, (3.6), and (3.5), we obtain

$$
\begin{aligned}
\int_{\mathbf{R}^{2}}\left\langle\partial_{s} \phi, \partial_{s} \phi\right\rangle_{\phi^{*} h} \rho^{2} d x= & \int_{\mathbf{R}^{2}}\left\langle\rho^{2} \partial_{s} \phi,\left(\phi^{*} \nabla\right)_{j} \partial_{j} \phi\right\rangle_{\phi^{*} h} d x \\
= & -\int_{\mathbf{R}^{2}}\left\langle\rho^{2}\left(\phi^{*} \nabla\right)_{j} \partial_{s} \phi, \partial_{j} \phi\right\rangle_{\phi^{*} h} \rho^{2} d x \\
& -2 \int_{\mathbf{R}^{2}}\left\langle\rho \partial_{j} \rho \partial_{s} \phi, \partial_{j} \phi\right\rangle_{\phi^{*} h} d x \\
= & -\int_{\mathbf{R}^{2}}\left\langle\left(\phi^{*} \nabla\right)_{s} \partial_{j} \phi, \partial_{j} \phi\right\rangle_{\phi^{*} h} \rho^{2} d x \\
& -2 \int_{\mathbf{R}^{2}}\left\langle\rho \partial_{j} \rho \partial_{s} \phi, \partial_{j} \phi\right\rangle_{\phi^{*} h} d x \\
= & -\partial_{s} \int_{\mathbf{R}^{2}} \mathbf{e}_{1} \rho^{2} d x-2 \int_{\mathbf{R}^{2}}\left\langle\partial_{s} \phi, \partial_{j} \phi\right\rangle_{\phi^{*} h} \rho \cdot \partial_{j} \rho d x .
\end{aligned}
$$

By Cauchy-Schwarz we have

$$
\int_{\mathbf{R}^{2}}\left\langle\partial_{s} \phi, \partial_{s} \phi\right\rangle_{\phi^{*} h} \rho^{2} d x+\partial_{s} \int_{\mathbf{R}^{2}} \mathbf{e}_{1} \rho^{2} d x \lesssim \int_{\mathbf{R}^{2}}\left|\partial_{s} \phi\right|\left|\partial_{x} \phi\right|_{\phi^{*} h}\left|\partial_{x} \rho\right|_{\phi^{*} h} \rho d x,
$$

and so from Young's inequality it follows that

$$
\int_{\mathbf{R}^{2}}\left\langle\partial_{s} \phi, \partial_{s} \phi\right\rangle_{\phi^{*} h} \rho^{2} d x+\partial_{s} \int_{\mathbf{R}^{2}} \mathbf{e}_{1} \rho^{2} d x \leq \int_{\mathbf{R}^{2}}\left|\partial_{s} \phi\right|_{\phi^{*} h}^{2} \rho^{2} d x+\frac{C}{R^{2}} \int_{\mathbf{R}^{2}}\left|\partial_{x} \phi\right|_{\phi^{*} h}^{2} d x .
$$


Therefore, in view of the smoothness of $\phi$ and the fact that $\rho$ has compact support, it holds that

$$
\partial_{s} \int_{\mathbf{R}^{2}} \mathbf{e}_{1} \rho^{2} d x \lesssim R^{-2} \int_{\mathbf{R}^{2}} \mathbf{e}_{1} d x .
$$

Integrating in (5.6), we conclude

$$
\begin{aligned}
E(\phi(s) 1 B(x, R)) & \leq \frac{1}{2} \int_{\mathbf{R}^{2}} \mathbf{e}_{1}(s) \rho^{2} d x \\
& \leq \frac{1}{2} \int_{\mathbf{R}^{2}} \mathbf{e}_{1}(0) \rho^{2} d x+\frac{C}{R^{2}} \int_{0}^{s} \int_{\mathbf{R}^{2}} \mathbf{e}_{1}\left(s^{\prime}\right) d x d s^{\prime} \\
& \leq E(\phi(0) 1 B(x, 2 R))+C \frac{s}{R^{2}} E_{0} .
\end{aligned}
$$

\section{Minimal Blowup solutions}

Recall that the ground state energy $E_{\text {crit }}$ is defined to be equal to the minimum energy carried by a finite energy nontrivial harmonic map $\mathbf{R}^{2} \rightarrow \mathcal{M}$ provided such maps exist and is set equal to $+\infty$ if such maps do not exist. Our goal is to show that there exists an energy-dependent constant $M$ such that

$$
\|\phi\|_{\mathcal{E}([0, \infty))} \leq M(E)<\infty
$$

whenever $E(\phi(0)) \leq E<E_{\text {crit }}$. It follows from interpolating between the small energy bounds (4.3) (with $k=1$ ) and (4.5) (with $k=1$ ) of Theorem 4.2 that such an inequality holds for $E$ sufficiently small. Let $E^{*}$ be the supremum of $\tilde{E}$ for which $\|\phi\|_{\mathcal{E}[0, \infty)} \leq M(\tilde{E})<\infty$ whenever $E(\phi(0)) \leq \tilde{E}$.

Proposition 6.1. It holds that

$$
E^{*}=E_{\text {crit }} .
$$

Proof. The proof will proceed by contradiction. It is clear that necessarily $E^{*} \leq E_{\text {crit }}$, because nontrivial harmonic maps as stationary solutions cannot satisfy (6.1). Hence we shall assume $E^{*}<E_{\text {crit }}$. By taking a family of initial data with energy strictly between $E^{*}$ and $E_{\text {crit }}$ and tending to $E^{*}$, we may extract along some subsequence and in a suitably strong sense a nontrivial harmonic map. Crucial use is made of the definition of $E^{*}$ in order to establish this. However, as energy is nonincreasing along the flow, the heat evolution $\phi$ always has energy less than and bounded away from the minimum energy $E_{\text {crit }}$ required to form a nontrivial harmonic map, which provides the contradiction. Nowhere must we assume that $E_{\text {crit }}$ is finite, and thus our argument is also valid in the case where $\mathcal{M}$ does not admit any finite energy harmonic maps. 
Let $\eta>0$ be a small parameter $\left(\eta \ll 1, E^{*}, E_{\text {crit }}\right)$. For any $\phi$ with classical initial data $\phi(0)$ having energy $E(\phi(0))<E_{\text {crit }}$, let $s^{\prime}=s^{\prime}(\phi)$ be the first time such that

$$
\|\phi\|_{\mathcal{E}\left(\left[0, s^{\prime}\right]\right)}=\eta
$$

If there is no such time $s^{\prime}$ or if $s^{\prime}=\infty$, then Lemma 4.13 is in force globally; therefore we may assume without loss of generality that the $s^{\prime}$ that we consider are finite.

Let $\epsilon>0$ be such that $\epsilon \ll \eta$. Suppose $E^{*}<E_{\text {crit }}$. If $E$ satisfies $E^{*}<E<$ $E_{\text {crit }}$ and yet $E\left(\phi\left(s^{\prime}\right)\right)<E^{*}-\epsilon$ independently of $\phi$, then there exists a bound of the form (6.1) for $E$, thus contradicting the choice of $E^{*}$. Therefore for all $n$ large there exists a heat flow $\phi_{n}$ with energy $E^{*}<E\left(\phi_{n}(0)\right)<E^{*}+1 / n<$ $E_{\text {crit }}$ such that

$$
E\left(\phi_{n}\left(s_{n}^{\prime}\right)\right)>E^{*}-\frac{1}{n} .
$$

Using scale invariance of the energy with respect to (1.3) we may assume that each $s_{n}^{\prime}=1$.

By (5.1) it follows that for all $R>0$

$$
\begin{aligned}
\eta^{4}=\left\|\phi_{n}\right\|_{\mathcal{E}[0,1]}^{4} \lesssim & \left\|E\left(\phi_{n} 1 B_{R}(x)\right)\right\|_{L_{s, x}^{\infty}\left([0,1] \times \mathbf{R}^{2}\right)} \times \\
& \times\left(\left\|\sqrt{\mathbf{e}_{2}}\right\|_{L_{s, x}^{2}\left([0,1] \times \mathbf{R}^{2}\right)}^{2}+\frac{1}{R^{2}}\left\|\sqrt{\mathbf{e}_{1}}\right\|_{L_{s, x}^{2}\left([0,1] \times \mathbf{R}^{2}\right)}^{2}\right) .
\end{aligned}
$$

Taking $k=1$ in (3.22), we have

$$
\mathbf{E}_{1}(1)-\mathbf{E}_{1}(0)+2\left\|\sqrt{\mathbf{e}_{2}}\right\|_{L_{s, x}^{2}\left([0,1] \times \mathbf{R}^{2}\right)}^{2} \lesssim\left\|\sqrt{\mathbf{e}_{1}}\right\|_{L_{s, x}^{4}\left([0,1] \times \mathbf{R}^{2}\right)}^{4}
$$

For large $n, E\left(\phi_{n}(1)\right) \sim E^{*}$ by construction and therefore from (6.3) it follows that $\left\|\sqrt{\mathbf{e}_{2}}\right\|_{L_{s}^{2} L_{x}^{2}\left([0,1] \times \mathbf{R}^{2}\right)}^{2} E_{E^{*}} \eta^{4}$. Using both of these facts in (6.2) (and suppressing now the dependence upon $E^{*}$ in subsequent inequalities) we get for $R>0$ that

$$
\eta^{4} \lesssim\left\|E\left(\phi_{n} 1 B_{R}(x)\right)\right\|_{L_{s, x}^{\infty}\left([0,1] \times \mathbf{R}^{2}\right)}\left(\eta^{4}+\frac{1}{R^{2}}\right) .
$$

For large $n$ pick $r_{n}>0$ so that $r_{n} \sim_{\eta} 1$ and

$$
1 \lesssim\left\|E\left(\phi_{n} 1 B_{r_{n}}(x)\right)\right\|_{L_{s, x}^{\infty}\left([0,1] \times \mathbf{R}^{2}\right)} .
$$

Thus for all large $n$ we may pick $x_{n} \in \mathbf{R}^{2}$ and $0 \leq s_{n} \leq 1$ so that

$$
1 \lesssim_{\eta} E\left(\phi\left(s_{n}\right) 1 B_{r_{n}}\left(x_{n}\right)\right)
$$

By (5.5) we have

$$
E\left(\phi_{n}\left(s_{n}\right) 1 B_{r_{n}}\left(x_{n}\right)\right) \leq E\left(\phi_{n}(s) 1 B_{2 r_{n}}\left(x_{n}\right)\right)+C \frac{s_{n}-s}{r_{n}^{2}} E\left(\phi_{n}(s)\right),
$$

for $0 \leq s \leq s_{n}$, which in view of our choice of $x_{n}, r_{n}$, and $s_{n}$, means

$$
1 \lesssim_{\eta} E\left(\phi_{n}(s) 1 B_{2 r_{n}}\left(x_{n}\right)\right)+C \frac{s_{n}-s}{r_{n}^{2}} E\left(\phi_{n}(s)\right) .
$$


As $r_{n} \sim 1$ and $0 \leq s_{n}-s \leq 1$, we may choose $\tilde{r}_{n} \geq r_{n}, \tilde{r}_{n} \sim_{\eta} r_{n}$ so that

$$
1 \lesssim \eta E\left(\phi_{n}(s) 1 B_{\tilde{r}_{n}}\left(x_{n}\right)\right)
$$

for all $0 \leq s \leq s_{n}$. Set

$$
\tilde{\phi}_{n}(s, x):=\phi_{n}\left(s \tilde{r}_{n}^{2}, x_{n}+x \tilde{r}_{n}\right) .
$$

Since $s_{n} / \tilde{r}_{n}^{2} \gtrsim 1$, we have for some sufficiently small constant $c>0$ that each $\tilde{\phi}_{n}$ is a heat flow with

$$
1 \lesssim \eta\left(\tilde{\phi}_{n}(s) 1 B(0,1)\right)
$$

for all $0 \leq s \leq c \eta$. Set $I=[0, c \eta]$. To each $\tilde{\phi}_{n}$ we have associated its tension field $\mathcal{T}_{n}$ given by

$$
\mathcal{T}_{n}:=\left(\tilde{\phi}_{n}^{*} \nabla\right)_{x} \partial_{x} \tilde{\phi}_{n}
$$

Then

$$
\left\|\mathcal{T}_{n}\right\|_{L_{s, x}^{2}\left(I \times \mathbf{R}^{2}\right)} \rightarrow 0 \quad \text { as } \quad n \rightarrow \infty
$$

since $E\left(\phi_{n}(1)\right)-E\left(\phi_{n}(0)\right) \rightarrow_{n \rightarrow \infty} 0$ (by construction) and because the energy functional is monotonic (Proposition 4.5). Passing to a subsequence if necessary, choose times $\tilde{s}_{n} \in I$ so that

$$
\left\|\mathcal{T}_{n}\left(\tilde{s}_{n}\right)\right\|_{L_{x}^{2}\left(\mathbf{R}^{2}\right)} \rightarrow 0
$$

Set $\psi_{n}(s):=\tilde{\phi}_{n}\left(s-\tilde{s}_{n}\right)$. Therefore the tension fields associated to $\psi_{n}$ satisfy

$$
\left\|\left(\psi_{n}^{*} \nabla\right)_{x} \partial_{x} \psi_{n}(0)\right\|_{L_{x}^{2}\left(\mathbf{R}^{2}\right)} \rightarrow 0
$$

and so in view of [20, Proposition 5.1] the $\psi_{n}(0)$ converge strongly along some subsequence in the Sobolev space $H_{x \text {, loc }}^{2}$ to a harmonic map $\psi_{0}$ away from any "energy bubbles", which asymptotically have a harmonic map profile. As $E\left(\psi_{n}(0)\right)<E_{\text {crit }}-\epsilon^{\prime}$ uniformly in $n$ for some $\epsilon^{\prime}>0$, it follows that energy bubble formation is impossible and that $\psi_{0}$ must be a trivial harmonic map. This however is precluded by the fact that $E\left(\psi_{n}(0) 1 B(0,1)\right) \gtrsim_{\eta} 1$ uniformly in $n$. From this contradiction we conclude that $E^{*}=E_{\text {crit }}$.

Combining Lemma 4.13 and Theorem 4.1, we conclude from $E^{*}=E_{\text {crit }}$ the following

Theorem 6.2. For any classical initial data $\phi_{0}$ with $E\left(\phi_{0}\right)<E_{\text {crit }}$ we have that there exists a unique global smooth heat flow $\phi$ with initial data $\phi_{0}$. 
Moreover, $\phi$ satisfies the estimates

$$
\begin{array}{r}
\int_{0}^{\infty} \int_{\mathbf{R}^{2}} s^{k-1} \mathbf{e}_{k+1}(s, x) d x d s \lesssim E_{0}, k \\
\sup _{0<s<\infty} s^{k-1} \int_{\mathbf{R}^{2}} \mathbf{e}_{k}(s, x) d x \\
\sup _{\substack{0<s<\infty \\
x \in \mathbf{R}^{2}}} s^{k} \mathbf{e}_{k}(s, x) \lesssim_{E_{0}, k} 1, \\
\int_{0}^{\infty} s^{k-1} \sup _{x \in \mathbf{R}^{2}} \mathbf{e}_{k}(s, x) d s \lesssim_{E_{0}, k} 1 .
\end{array}
$$

for each $k \geq 1$.

Corollary 6.3. Let $\phi_{0}, \phi$ be as in Theorem 6.2, classical with respect to $\phi(\infty) \in \mathcal{M}$. Then for each fixed $s$ the function $\phi(s)$ is Schwartz with respect to $\phi(\infty)$. Additionally, $\phi(s)$ converges as $s \rightarrow \infty$ in the $C_{x}^{\infty}\left(\mathbf{R}^{2} \rightarrow \mathbf{R}^{+} \times \mathcal{M}\right)$ topology to $\phi(\infty)$.

Proof. By Theorems 4.1 and 6.2 and a simple continuity argument we have that $\phi(s)-\phi(\infty)$ is Schwartz for each fixed $s \geq 0$.

Energy bounds imply $\phi(s, x) \rightarrow_{s \rightarrow \infty} \phi(\infty)$ in the uniform topology and that $\partial_{x}^{k} \phi(s, x) \rightarrow_{s \rightarrow \infty} 0$ in the uniform topology for all integers $k \geq 1$. Therefore, $\phi(s)$ converges to $\phi(\infty)$ in $C_{x}^{\infty}\left(\mathbf{R}^{2} \rightarrow \mathcal{M}\right)$ as $s \rightarrow \infty$. To upgrade this to convergence in $C_{x}^{\infty}\left(\mathbf{R}^{2} \rightarrow \mathbf{R}^{+} \times \mathcal{M}\right)$, use (1.4) to convert time derivatives into spatial derivatives.

\section{The CALORIC GAUGE}

For simplicity, we split this section into two parts, in the first ignoring the time variable $t$, and in the second taking it up again.

7.1. The static caloric gauge. Let $\phi: \mathbf{R}^{+} \times \mathbf{R}^{2} \rightarrow \mathcal{M}$ be a heat flow with classical initial data $\phi_{0}$ that equals $\phi(\infty)$ at spatial infinity. We now introduce the notion of a gauge, followed by the caloric gauge which originates in [23]. Our conventions and presentation draw on [23], 26], and [24, Chapter 6]. For a thorough introduction to gauges (sometimes referred to as moving frames), see [19, Chapter 7].

Given any $p \in \mathcal{M}$ we define an orthonormal frame at $p$ to be any orthogonal orientation-preserving map $e: \mathbf{R}^{m} \rightarrow T_{p} \mathcal{M}$ from $\mathbf{R}^{m}$ to the tangent space $T_{p} \mathcal{M}$ at $p$. We let $\operatorname{Frame}\left(T_{p} \mathcal{M}\right)$ stand for the space of all such frames and note that this space admits the obvious transitive action of the special orthogonal group $S O(m)$. We define the orthonormal frame bundle Frame $\left(\phi^{*} T \mathcal{M}\right)$ of $\phi$ to be the space of all pairs $((s, x), e)$ where $(s, x) \in$ $\mathbf{R}^{+} \times \mathbf{R}^{2}$ and $e \in \operatorname{Frame}\left(T_{\phi(s, x)} \mathcal{M}\right)$. This is a fiber bundle over $\mathbf{R}^{+} \times \mathbf{R}^{2}$. 
We define an orthonormal frame $e \in \Gamma\left(\operatorname{Frame}\left(\phi^{*} T \mathcal{M}\right)\right)$ for $\phi$ to be a section of Frame $\left(\phi^{*} T \mathcal{M}\right)$, i.e., a smooth assignment $e(s, x) \in \operatorname{Frame}\left(T_{\phi(s, x)} \mathcal{M}\right)$ of an orthonormal frame at $\phi(s, x)$ to every point $(s, x) \in \mathbf{R}^{+} \times \mathbf{R}^{2}$.

Each orthonormal frame $e \in \Gamma\left(\operatorname{Frame}\left(\phi^{*} T \mathcal{M}\right)\right)$ provides an orthogonal, orientation-preserving identification between the vector bundle $\phi^{*} T \mathcal{M}$ bearing the metric $\phi^{*} h$ and the trivial bundle $\left(\mathbf{R}^{+} \times \mathbf{R}^{2}\right) \times \mathbf{R}^{m}$ bearing the Euclidean metric on $\mathbf{R}^{m}$. Hence via $e$ we may pull back sections $\Psi \in \Gamma\left(\phi^{*} T \mathcal{M}\right)$ to functions $e^{*} \Psi$ and we may pull back the connection $\phi^{*} \nabla$ on $\phi^{*} T \mathcal{M}$ to a connection $D$ on the trivial bundle $\left(\mathbf{R}^{+} \times \mathbf{R}^{2}\right) \times \mathbf{R}^{m}$ so that

$$
D_{\alpha}:=\partial_{\alpha}+A_{\alpha}
$$

with $A_{\alpha} \in \mathfrak{s o}(m)$ the skew-adjoint $m \times m$ matrix field defined by

$$
\left(A_{\alpha}\right)_{a b}:=\left\langle\left(\phi^{*} \nabla\right)_{\alpha} e_{a}, e_{b}\right\rangle_{\phi^{*} h}
$$

and $e_{1}, \ldots, e_{m}$ the images under $e$ of the standard orthonormal basis vectors of $\mathbf{R}^{m}$.

Throughout this section Greek lettered indices denote arbitrary variables, i.e. space or time, whereas Roman lettered indices are only used to denote spatial variables.

Let us define the derivative fields $\psi_{\alpha}: \mathbf{R}^{+} \times \mathbf{R}^{2} \rightarrow \mathbf{R}^{m}$ by

$$
\psi_{\alpha}:=e^{*} \partial_{\alpha} \phi
$$

For short we write $\psi_{x}=\left(\psi_{1}, \psi_{2}\right)$ and $A_{x}=\left(A_{1}, A_{2}\right)$. The zero-torsion property (3.6) now manifests itself as

$$
D_{\alpha} \psi_{\beta}=D_{\beta} \psi_{\alpha}
$$

which equivalently may be expressed as

$$
\partial_{\alpha} \psi_{\beta}-\partial_{\beta} \psi_{\alpha}=A_{\beta} \psi_{\alpha}-A_{\alpha} \psi_{\beta}
$$

The Leibnitz rule (3.5) becomes

$$
\partial_{\alpha}\left(\psi \cdot \psi^{\prime}\right)=\left(D_{\alpha} \psi\right) \cdot \psi^{\prime}+\psi \cdot\left(D_{\alpha} \cdot \psi^{\prime}\right) .
$$

Note that the covariant derivative $D_{x}$ acts on matrix fields $B$ via

$$
D_{x} B=\partial_{x} B+[A, B] .
$$

Curvature in this context is given by

$$
F_{\alpha \beta}:=\left[D_{\alpha}, D_{\beta}\right]=\partial_{\alpha} A_{\beta}-\partial_{\beta} A_{\alpha}+\left[A_{\alpha}, A_{\beta}\right] .
$$

Using the coordinate expression

$$
\left(\phi^{*} \nabla\right)_{\alpha}\left(\phi^{*} \nabla\right)_{\beta} \Psi^{c}-\left(\phi^{*} \nabla\right)_{\beta}\left(\phi^{*} \nabla\right)_{\alpha} \Psi^{c}=\left(\partial_{\alpha} \phi\right)^{a}\left(\partial_{\beta} \phi\right)^{b} \mathcal{R}_{a b d}^{c} \Psi^{d},
$$

for curvature, we can write

$$
F_{\alpha \beta}=\left(e^{*} \mathcal{R}(\phi)\right)\left(\psi_{\alpha}, \psi_{\beta}\right)
$$


where $\left(e^{*} \mathcal{R}(\phi)\right)(\cdot, \cdot)$ denotes the pullback of the Riemannian curvature tensor $\mathcal{R}$ on $\mathcal{M}$, defined in local coordinates via

$$
\left(e\left(e^{*} \mathcal{R}(\phi)\right)\left(\psi_{\alpha}, \psi_{\beta}\right) \phi\right)^{c}=\mathcal{R}_{a b d}^{c}(\phi)\left(e \psi_{\alpha}\right)^{a}\left(e \psi_{\beta}\right)^{b}(e \phi)^{d} .
$$

In terms of the frame $e$ the heat flow equation (1.1) becomes

$$
\psi_{s}=D_{j} \psi_{j}
$$

Note the gauge symmetry inherent in (7.8): It is invariant under the transformation

$$
\begin{aligned}
& \phi \mapsto \phi ; \quad \quad \mapsto e U ; \quad \psi_{\alpha} \mapsto U^{-1} \psi_{\alpha} ; \\
& D_{\alpha} \mapsto U^{-1} D_{\alpha} U ; \quad A_{\alpha} \mapsto U^{-1} \partial_{\alpha} U+U^{-1} A_{\alpha} U,
\end{aligned}
$$

for any choice of gauge transform $U: \mathbf{R}^{+} \times \mathbf{R}^{2} \rightarrow S O(m)$.

We now introduce the caloric gauge, defining it as in [26]:

Definition 7.1. Let $\phi_{0}: \mathbf{R}^{2} \rightarrow \mathcal{M}$ be a function Schwartz with respect to $\phi(\infty) \in \mathcal{M}$ and with energy $E_{0}$ less than $E_{\text {crit }}$. Let $\phi: \mathbf{R}^{+} \times \mathbf{R}^{2} \rightarrow \mathcal{M}$ be its heat flow extension. We say that a gauge $e$ is a caloric gauge for $\phi$ with boundary frame $e(\infty) \in \operatorname{Frame}\left(T_{\phi(\infty)} \mathcal{M}\right)$ if we have

$$
A_{s}=0
$$

throughout $\mathbf{R}^{+} \times \mathbf{R}^{2}$ and also

$$
\lim _{s \rightarrow \infty} e(s, x)=e(\infty)
$$

for all $x \in \mathbf{R}^{2}$.

Theorem 7.2 (Existence and uniqueness of caloric gauge). Let $\phi: \mathbf{R}^{+} \times$ $\mathbf{R}^{2} \rightarrow \mathcal{M}$ be a heat flow with classical initial data that equals $\phi(\infty) \in \mathcal{M}$ at infinity and that has initial energy $E_{0}$ less than $E_{\text {crit }}$. Let $e(\infty) \in$ Frame $\left(T_{\phi(\infty)} \mathcal{M}\right)$ be an orthonormal frame at $\phi(\infty)$. Then there exists a unique caloric gauge e $\in \Gamma\left(\operatorname{Frame}\left(\phi^{*} T \mathcal{M}\right)\right)$ for $\phi$ with boundary frame $e(\infty)$. Furthermore, for each fixed $s$, the $\psi_{\alpha}$ are Schwartz functions in space.

Proof. The caloric gauge condition $A_{s}=0$ implies that the frame $e$ evolves according to

$$
\left(\phi^{*} \nabla\right)_{s} e_{j}=0 .
$$

If $e, e^{\prime}$ are caloric gauges with the same boundary frame $e(\infty)$, then $\left|e_{j}-e_{j}^{\prime}\right|$ is constant in $s$. Uniqueness follows since $\left|e_{j}-e_{j}^{\prime}\right|$ vanishes as $s \rightarrow \infty$.

Turning now to existence, given initial data $\phi_{0}$, choose an arbitrary smooth orthonormal frame $e(0, x) \in \operatorname{Frame}\left(T_{\phi(0, x)} \mathcal{M}\right)$ that differs from a constant frame by a Schwartz function. This is possible because we assume that the initial data $\phi_{0}$ is classical and because the spatial domain $\mathbf{R}^{2}$ is contractible. As $\phi^{*} \nabla$ respects the metric on $\phi^{*} T \mathcal{M}$, we have from $(7.9)$ that $e$ remains orthonormal as it evolves in $s$. It follows from the Picard theorem that $e$ 
may be defined globally. Smoothness of $\phi$ and $e(0, \cdot)$ implies that $e, \psi$, and $A$ are all smooth.

Viewing the energy density estimates (6.6) in terms of frames yields

$$
\left|D_{x}^{k-1} \psi_{x}\right| \lesssim_{E_{0}, k} s^{-k / 2}
$$

for $k \geq 1$. Using (7.8), we also have for $k \geq 1$ that

$$
\left|D_{x}^{k-1} \psi_{s}\right| \lesssim_{E_{0}, k} s^{-(k+1) / 2} .
$$

Combining $A_{s}=0$ and (7.6) gives

$$
\partial_{s} A_{x}=F_{x s}
$$

Therefore, using (7.7), tensoriality, and bounded geometry, we have

$$
\left|\partial_{s} D_{x}^{k} A_{x}\right| \lesssim k \sum_{\substack{j_{1}+j_{2}+\cdots j_{\ell}=k \\ j_{1}, j_{2}, \ldots, j_{\ell} \geq 0 \\ \ell=2, \ldots, k+2}}\left|D_{x}^{j_{1}} \psi_{s}\right|\left|D_{x}^{j_{2}} \psi_{x}\right| \cdots\left|D_{x}^{j_{\ell}} \psi_{x}\right|
$$

for $k \geq 0$. Using (7.10) and (7.11) in (7.13) implies

$$
\left|\partial_{s} D_{x}^{k} A_{x}\right| \lesssim_{k} s^{-(k+3) / 2} \text {. }
$$

Therefore, since $A_{x}$ and its derivatives are bounded at $s=0$,

$$
\left|D_{x}^{k} A_{x}\right| \lesssim_{k, \phi} 1
$$

on $\mathbf{R}^{+} \times \mathbf{R}^{2}$ for all $k \geq 0$, which, combined with (17.5) and the triangle inequality, implies

$$
\left|\partial_{x}^{k} A_{x}\right| \lesssim_{k, \phi} 1
$$

Using (7.14) and (7.15) in (17.10) and (7.11) leads to

$$
\left|\partial_{x}^{k} \psi_{x}\right| \lesssim_{k} s^{-1 / 2} \text { and }\left|\partial_{x}^{k} \psi_{s}\right| \lesssim_{k} s^{-1} \text {. }
$$

In view of (7.12), differentiating $F_{\alpha \beta}=\left(e^{*} R(\phi)\right)\left(\psi_{\alpha}, \psi_{\beta}\right)$ and using (7.14), (7.15), (7.10), (7.11), and bounded geometry, we conclude $A_{x}(s)$ converges in $C_{x}^{\infty}\left(\mathbf{R}^{2} \rightarrow \mathfrak{s o}(m)\right)$ to some limit $A_{x}(\infty)$ as $s \rightarrow \infty$. Because of this convergence and the fact that $\phi(s)$ is spatially Schwartz uniformly in $s$, it follows from definition (7.2) of the connection coefficients $\left(A_{\alpha}\right)_{a b}$ that $\iota \circ e$, viewed as a linear transformation, is locally bounded in $C_{x}^{\infty}$ uniformly in $s$.

From (2.9) and (1.4) it follows that

$$
\left\|\partial_{s} \phi(s)\right\|_{L_{x}^{\infty}} \leq\left\|e^{s \Delta} \phi_{0}\right\|_{L_{x}^{\infty}}+\int_{0}^{s}\left\|e^{\left(s-s^{\prime}\right) \Delta}\left(\Pi(\phi)\left(\partial_{x} \phi, \partial_{x} \phi\right)\right)\left(s^{\prime}\right)\right\|_{L_{x}^{\infty}} d s^{\prime},
$$

wherein $e^{s \Delta}$ retains its meaning given by (2.4) as is clear from context (as opposed to referring to the caloric gauge $e$ ). From Young's inequality and the fact that $\phi(s)$ is spatially Schwartz in $x$ uniformly in $s$, we conclude

$$
\int_{0}^{\infty}\left\|\partial_{s} \phi(s, \cdot)\right\|_{L_{x}^{\infty}} d s \lesssim_{\phi} 1
$$


which, upon integrating in (7.9), establishes uniform convergence of $e(s, \cdot)$ to some limit $e(\infty, \cdot)$. Using the local $C^{\infty}$ bounds for $\iota \circ e$ upgrades this to convergence in $C_{x, \text { loc }}^{\infty}$, thereby showing $e(\infty, \cdot)$ is smooth. Taking limits in the definition (7.2) of $A$ shows that indeed $A_{x}(\infty, \cdot)$ are the connection coefficients for the frame $e(\infty, \cdot)$.

Applying a smooth gauge transformation $U(s, x)=U(x)$ to normalize $e(\infty, \cdot)=$ $e(\infty)$, we get the caloric gauge. In this gauge $A_{x}(s)$ converges in $C_{\text {loc }}^{\infty}\left(\mathbf{R}^{2} \rightarrow\right.$ $\mathfrak{s o}(m))$ to zero as $s \rightarrow \infty$. In particular, this normalization preserves the caloric gauge condition $A_{s}=0$ and specifies the boundary frame $e(\infty)$.

It remains to show that the $\psi_{\alpha}$ are Schwartz. This is an easy consequence of Corollary 7.5 stated below.

Lemma 7.3. (Equations of motion). Let $\phi: \mathbf{R}^{+} \times \mathbf{R}^{2} \rightarrow \mathcal{M}$ be a heat flow with classical initial data whose energy $E_{0}$ is less than $E_{\text {crit }}$, let $e$ be a caloric gauge for $\phi$, and let $\psi_{x}, \psi_{s}, A_{x}$ be the associated derivative fields and connection fields. Then we have the evolution equations

$$
\begin{aligned}
& \partial_{s} \psi_{x}=D_{x} \psi_{s}=\partial_{x} \psi_{s}+A_{x} \psi_{s} \\
& \partial_{s} A_{x}=F_{x s} \\
& \partial_{s} \psi_{\alpha}=D_{i} D_{i} \psi_{\alpha}+F_{\alpha i} .
\end{aligned}
$$

Proof. The first equation (7.17) follows from $A_{s}=0$, the zero-torsion property (7.4), and (7.1).

As already noted previously (see (7.12) $),(7.18)$ is a consequence of $A_{s}=0$ and (7.6).

To prove (7.19), we use $A_{s}=0$ and sequentially apply (7.4), (17.8), (17.6), and (7.4):

$$
\begin{aligned}
\partial_{s} \psi_{\alpha} & =D_{\alpha} \psi_{s} \\
& =D_{\alpha} D_{i} \psi_{i} \\
& =D_{i} D_{\alpha} \psi_{i}+F_{\alpha i} \\
& =D_{i} D_{i} \psi_{\alpha}+F_{\alpha i} .
\end{aligned}
$$

Proposition 7.4. (Connection bounds). Let $\phi$ be a heat flow with classical initial data whose energy $E_{0}$ is less than $E_{\text {crit }}$. Let e be a caloric gauge for $\phi$, and let $A_{x}$ denote the connection fields. Then we have the pointwise bounds

$$
\begin{aligned}
&\left\|\partial_{x}^{k} A_{x}(s)\right\|_{L_{x}^{\infty}\left(\mathbf{R}^{2}\right)} \lesssim E_{0}, k \\
&\left\|s^{k} A_{x}(s)\right\|_{L_{x}^{2}\left(\mathbf{R}^{2}\right)} \lesssim E_{0}, k \\
& \|{ }^{-k / 2} / 2
\end{aligned}
$$


for all $k \geq 0$ and $s>0$, and the integrated estimates

$$
\begin{gathered}
\int_{0}^{\infty} s^{(k-1) / 2}\left\|\partial_{x}^{k} A_{x}(s)\right\|_{L_{x}^{\infty}\left(\mathbf{R}^{2}\right)} d s \lesssim_{E_{0}, k} 1, \\
\int_{0}^{\infty} s^{(k-1) / 2}\left\|\partial_{x}^{k+1} A_{x}(s)\right\|_{L_{x}^{2}\left(\mathbf{R}^{2}\right)} d s \lesssim_{E_{0}, k} 1
\end{gathered}
$$

for all $k \geq 0$.

Proof. From (7.12) we have the integral representation

$$
A_{x}(s)=\int_{s}^{\infty} F_{s x}\left(s^{\prime}\right) d s^{\prime}
$$

Differentiating covariantly as in (7.13), we get

$$
\left|D_{x}^{k} A_{x}(s)\right| \lesssim \int_{s}^{\infty} \sum_{\substack{j_{1}+j_{2}+\cdots j_{\ell}=k+3 \\ j_{1}, j_{j}, \ldots, j_{\ell} \geq 0 \\ \ell=2, \ldots, k+2}} \mathbf{e}_{j_{1}}^{1 / 2}\left(s^{\prime}\right) \cdots \mathbf{e}_{j_{\ell}}^{1 / 2}\left(s^{\prime}\right) d s^{\prime}
$$

and so applying (6.6) yields

$$
\left\|D_{x}^{k} A_{x}(s)\right\|_{L_{x}^{\infty}\left(\mathbf{R}^{2}\right)} \lesssim_{k} s^{-(k+1) / 2}
$$

for $k \geq 0$. Together (7.5) and an inductive argument prove (7.20).

Applying Minkowski's and Hölder's inequalities in (7.24), we arrive at

$$
\begin{aligned}
\left\|D_{x}^{k} A_{x}(s)\right\|_{L_{x}^{2}} \lesssim \int_{s}^{\infty} \sum_{\substack{j_{1}+j_{2}+\cdots, j_{\ell}=k \\
j_{1}, j_{2}, \ldots, j_{\ell} \geq 0 \\
\ell=2, \ldots, k+2}}\left\|\mathbf{e}_{j_{1}+2}^{1 / 2}\left(s^{\prime}\right)\right\|_{L_{x}^{2}}\left\|\mathbf{e}_{j_{2}+1}^{1 / 2}\left(s^{\prime}\right)\right\|_{L_{x}^{\infty}} \\
\quad \times\left\|\mathbf{e}_{j_{3}}^{1 / 2}\left(s^{\prime}\right)\right\|_{L_{x}^{\infty}} \cdots\left\|\mathbf{e}_{j_{\ell}}^{1 / 2}\left(s^{\prime}\right)\right\|_{L_{x}^{\infty}} d s^{\prime} .
\end{aligned}
$$

Using (6.4), (6.7), and Cauchy-Schwarz leads to (7.21) with ordinary derivatives $\partial_{x}$ replaced by covariant derivatives $D_{x}$. Ordinary derivatives may be recovered using (7.5) and (7.20).

Using (7.25) with $k+1$ in place of $k$ and applying the arithmetic- geometricmean inequality yields

$$
\begin{aligned}
\left\|D_{x}^{k+1} A_{x}(s)\right\|_{L_{x}^{2}} \lesssim & \sum_{\substack{j_{1}+j_{2}+\cdots, j_{\ell}=k \\
j_{1}, j_{2}, \ldots, j_{\ell} \geq 0 \\
\ell=2, \ldots, k+2}} \int_{s}^{\infty}\left(s^{\prime}\right)^{j_{1}-1 / 2}\left\|\mathbf{e}_{j+2}^{1 / 2}\left(s^{\prime}\right)\right\|_{L_{x}^{2}}^{2} \\
& +\left(s^{\prime}\right)^{-j_{1}+1 / 2}\left\|\mathbf{e}_{j_{2}+1}^{1 / 2}\left(s^{\prime}\right)\right\|_{L_{x}^{\infty}}^{2}\left\|\mathbf{e}_{j_{3}}^{1 / 2}\left(s^{\prime}\right)\right\|_{L_{x}^{\infty}}^{2} \cdots\left\|\mathbf{e}_{j_{\ell}}^{1 / 2}\left(s^{\prime}\right)\right\|_{L_{x}^{\infty}}^{2} d s^{\prime},
\end{aligned}
$$

and hence by using (6.4) and Fubini on the first term of the right hand side and (6.6), (6.7), and Fubini on the second it follows that inequality (7.23) holds with ordinary derivatives replaced by covariant derivatives. To recover ordinary derivatives in (7.23), again use (7.5) and (7.20). 
Finally, note that if $k \geq 1$, then applying the Gagliardo-Nirenberg inequality (2.1) to (7.23) gives (7.22). Therefore it only remains to check the $k=0$ case: Using (7.24) and Fubini yields

$$
\int_{0}^{\infty} s^{-1 / 2}\left\|\partial_{x} A_{x}(s)\right\|_{L_{x}^{2}} d s \lesssim \int_{0}^{\infty} s^{-1 / 2}\left\|\mathbf{e}_{2}^{1 / 2}(s)\right\|_{L_{x}^{\infty}}\left\|\mathbf{e}_{1}^{1 / 2}(s)\right\|_{L_{x}^{\infty}} d s,
$$

and so the inequality follows from Cauchy-Schwarz and (6.7).

Corollary 7.5. Let $\phi$ be a heat flow with classical initial data with energy $E_{0}$ less than $E_{\text {crit }}$. Let e be a caloric gauge for $\phi$. Then

$$
\begin{array}{r}
\int_{0}^{\infty} s^{k-1}\left\|\partial_{x}^{k} \psi_{x}\right\|_{L_{x}^{2}\left(\mathbf{R}^{2}\right)}^{2} d s \lesssim E_{0}, k \\
\sup _{s>0} s^{(k-1) / 2}\left\|\partial_{x}^{k-1} \psi_{x}\right\|_{L_{x}^{2}\left(\mathbf{R}^{2}\right)} \lesssim_{E_{0}, k} 1, \\
\int_{0}^{\infty} s^{k-1}\left\|\partial_{x}^{k-1} \psi_{x}\right\|_{L_{x}^{\infty}\left(\mathbf{R}^{2}\right)}^{2} d s \lesssim_{E_{0}, k} 1, \\
\sup _{s>0} s^{k / 2}\left\|\partial_{x}^{k-1} \psi_{x}\right\|_{L_{x}^{\infty}\left(\mathbf{R}^{2}\right)} \lesssim_{E_{0}, k} 1
\end{array}
$$

for all $k \geq 1$. Analogous estimates hold if one replaces $\partial_{x} \psi_{x}$ with $\psi_{s}, \partial_{x}^{2}$ with $\partial_{s}$ and/or $\partial_{x}$ with $D_{x}$.

Proof. This follows from Theorem6.2, (7.20), and writing $\partial_{x}=D_{x}-A_{x}$.

\subsection{The dynamic caloric gauge.}

Theorem 7.6. (Dynamic caloric gauge). Let I be a time interval, let $\phi$ : $\mathbf{R}^{2} \times I \rightarrow \mathcal{M}$ be a smooth map Schwartz on each time slice with respect to the point $\phi(\infty) \in \mathcal{M}$, and let $e(\infty) \in \operatorname{Frame}\left(T_{\phi(\infty)} \mathcal{M}\right)$ be a frame for $\phi(\infty)$. Assume moreover that there is some $\epsilon>0$ so that $E(\phi(t))<E_{\text {crit }}-\epsilon$ for all $t \in I$.

Then we have the following conclusions. The map $\phi$ extends smoothly to a dynamic heat flow $\phi: \mathbf{R}^{+} \times \mathbf{R}^{2} \times I \rightarrow \mathcal{M}$ and converges to $\phi(\infty)$ in $C_{\mathrm{loc}}^{\infty}\left(\mathbf{R}^{2} \times\right.$ I) as $s \rightarrow \infty$. There exists a unique smooth frame $e \in \Gamma\left(\operatorname{Frame}\left(\phi^{*} T \mathcal{M}\right)\right)$ such that $e(t)$ is a caloric gauge for $\phi(t)$ that equals $e(\infty)$ at infinity for each $t \in I$. The map $\phi$ is Schwartz with respect to $\phi(\infty)$ and all derivatives of $\phi$ in $s, x, t$ are Schwartz in $x$ for each fixed $s, t$. Moreover, the time derivative field $\psi_{t}$ obeys

$$
\partial_{s} \psi_{t}=D_{i} D_{i} \psi_{t}+F_{t i}
$$

and the time connection field $A_{t}$ obeys

$$
\partial_{s} A_{t}=F_{t s} .
$$


Proof. The proof of uniqueness is as in Theorem [7.2. Without loss of generality we take $I$ compact. For each fixed $t$ we can extend the initial data $\phi(\cdot, t): \mathbf{R}^{2} \rightarrow \mathcal{M}$ to a smooth heat flow $\phi(\cdot, \cdot, t): \mathbf{R}^{+} \times \mathbf{R}^{2} \rightarrow \mathcal{M}$ by Theorem 6.2. As the global solution provided by Theorem 6.2 coincides with that directly provided locally by Picard iteration, the extension $\phi$ depends smoothly on the initial data since the nonlinearity being iterated is a smooth function of $\phi$. Noting that the constants in the bounds in Theorem 6.2 depend only upon the initial energy of the map and recalling that we assume $E(\phi(0, \cdot, t))$ is less than and bounded away from $E_{\text {crit }}$ uniformly in $t$, we have that $t \mapsto \phi(\cdot, \cdot, t)$ is locally smooth in smooth topologies, and therefore the fixed-time heat flows $\phi(\cdot, \cdot, t)$ can be joined together to create a smooth dynamic heat flow $\phi: \mathbf{R}^{+} \times \mathbf{R}^{2} \times I \rightarrow \mathcal{M}$. Uniform bounds in the limit follow from the bounds in Theorem 6.2. We note that in order to show that derivatives of $\phi$ involving the $t$ variable are Schwartz in $x$ for fixed $s, t$, it suffices to directly apply a Picard iteration scheme as in Theorem 4.1 .

The rest of the construction of the caloric gauge follows that in Theorem 7.2. except that now we must take into account the dynamic variable $t$ and associated fields $\psi_{t}, A_{t}$. In particular, the proofs of (7.26) and (7.27) are analogous to those of (7.19) and (7.18) of Lemma 7.3.

Acknowledgments. The author is indebted to Terence Tao for his encouragement, support, and guidance, and thanks Peter Petersen for his teaching and discussions. The author would also like to thank the anonymous referees for corrections and suggestions, and for pointing out in an earlier draft the need for an additional argument to handle the noncompact case.

\section{Appendix A. Heat Flow into nONCOMPACT MANifoldS}

We claimed that the caloric gauge construction is valid for any bounded geometry manifold $\mathcal{M}$, even though for convenience we made an additional technical assumption. In particular, we assumed that $\mathcal{M}$ was a closed manifold, i.e., compact and without boundary. Here we discuss how to remove this technical assumption.

First let us introduce an intrinsic notion of a Schwartz function from $\mathbf{R}^{2} \rightarrow$ $\mathcal{M}$. As discussed in $\$ \mathbb{1}$, we must choose a base point $p \in \mathcal{M}$ to which our functions shall decay since there is no such natural choice on a general manifold; however, once this point is selected, there is the following natural intrinsic definition of a Schwartz function.

Definition A.1. Let $\mathcal{M}$ be a manifold of bounded geometry and let $p \in \mathcal{M}$. A function $\phi: \mathbf{R}^{2} \rightarrow \mathcal{M}$ is said to be Schwartz with respect to $p$ provided $\phi \in C^{\infty}\left(\mathbf{R}^{2} \rightarrow \mathcal{M}\right)$ and provided $\phi$ satisfies the decay conditions

$$
d_{\mathcal{M}}(\phi(x), p)=O_{\phi, N}\left(\langle x\rangle^{-N}\right)
$$


for all positive integers $N$ and

$$
\left(\phi^{*} \nabla\right)_{x}^{\alpha} \phi(x)=O_{\phi, \alpha, N}\left(\langle x\rangle^{-N}\right)
$$

for all (nonzero) multiindices $\alpha$ and positive integers $N$.

When $\mathcal{M}$ is a compact manifold, this definition is equivalent to that given in 91. This is shown with a direct argument by choosing an smooth isometric embedding $\iota: \mathcal{M} \hookrightarrow \mathbf{R}^{n}$.

Equipped with a suitable definition of Schwartz function, we now give the underlying idea in moving from the compact case to the noncompact case, due to [8]. Start with Schwartz initial data $\phi_{0}: \mathbf{R}^{2} \rightarrow \mathcal{M}$. In view of the fast-decay of $\phi_{0}$ and the fact that it is smooth, it follows that the image of $\mathbf{R}^{2}$ under $\phi_{0}$ lies within a compact set of $\mathcal{M}$. One then sets up an embedding into a Euclidean space $\mathbf{R}^{n}$ to show local existence and proves that the heat flow $\phi$ generated from the initial data $\phi_{0}$ stays within a compact subset of $\mathcal{M}$ over its entire (maximal) interval of existence. The rigorous argument is found in [8, §3]. A posteriori, we choose a compact subset of $\mathcal{M}$, choose a smooth isometric embedding into some Euclidean space $\mathbf{R}^{n}$, and apply the Duhamel formula (4.1) as in $\$ 4$ in order to establish that the heat flow $\phi$ is extrinsically Schwartz. That the heat flow is also intrinsically Schwartz then follows from the fact that the flow has a precompact image in $\mathcal{M}$. The blowup criterion derived from the proof is the same as that for the compact case: the flow may be continued so long as $\partial_{x} \phi$ remains bounded.

We therefore have an entirely intrinsic analogue of Theorem 4.1, and, as indicated in [8, Remark 3.1], we know that there is a compact set in $\mathcal{M}$ in which the heat flow $\phi$ always remains.

We now discuss where, in addition to Theorem 4.1, compactness was exploited. Corollary 6.3 employs compactness explicitly in a very mild way, namely, to establish that smooth solutions are Schwartz. Based upon the proof of the corollary, it is clear that the intrinsic version of Theorem 4.1 suffices for this application.

A more subtle, implicit use of compactness is made in $\$ 6$, where the minimal blowup solution argument appeals to the energy quantization of harmonic maps (in particular, the existence of the groundstate energy $E_{\text {crit }}$ ) and to the bubbling profile result [20] of Struwe.

For noncompact target manifolds we slightly modify the usual definition of groundstate energy $E_{\text {crit }}$. Given a compact subset $K$ of $\mathcal{M}$, we may smoothly extend $K$ to a larger compact (Riemannian) manifold $\widetilde{K}$ without boundary, i.e., a closed manifold. Associate to the manifold $\widetilde{K}$ its groundstate energy $E_{\text {crit }}(\widetilde{K})$ (as defined in $§ 1$ ). Now define the groundstate energy of $K$ to be the supremum of energies $E_{\text {crit }}(\widetilde{K})$ taken over all smooth, closed extensions 
$\tilde{K}$ of $K$. Finally, set

$$
E_{\text {crit }}(\mathcal{M}):=\limsup _{n \rightarrow \infty} E_{\text {crit }}\left(K_{n}\right)
$$

where $K_{n}$ denotes a (nested) compact exhaustion of $\mathcal{M}$, i.e., each $K_{n}$ is compact, $K_{n} \subset K_{n+1}$, and $\cup_{n} K_{n}=\mathcal{M}$. To show that $E_{\text {crit }}(\mathcal{M})$ is welldefined, one may suitably interlace two given compact exhaustions $K_{n}, J_{m}$.

Lemma A.2. For any dimension $m$ we have

$$
E_{\text {crit }}\left(\mathbf{H}^{m}\right)=+\infty
$$

i.e., the ground state energy of hyperbolic space is infinite.

Proof. Any compact $K \subset \mathbf{H}^{m}$ can be smoothly extended to a closed manifold $\tilde{K}$ by means of taking the quotient of $\mathbf{H}^{m}$ by a suitable discrete group action. However, as shown in [5, §11], finite energy harmonic maps from $\mathbf{R}^{2} \rightarrow \mathbf{H}^{m}$ do not exist. Therefore $E_{\text {crit }}(\tilde{K})=+\infty$.

Now, given Schwartz initial data $\phi_{0}: \mathbf{R}^{2} \rightarrow \mathcal{M}$ with $E\left(\phi_{0}\right)<E_{\text {crit }}(\mathcal{M})$, we may choose a sufficiently large compact subset $K$ of $\mathcal{M}$ and suitable smooth closed manifold extension $\tilde{K}$ of $K$ so that $E\left(\phi_{0}\right)<E_{\text {crit }}(\tilde{K})$ and $\phi: I \times \mathbf{R}^{2} \rightarrow \mathcal{M}$ remains within $K$ for its entire interval of existence $I$. Replacing $\mathcal{M}$ with $\tilde{K}$, i.e., considering now $\phi$ as a heat flow into $\tilde{K}$, we may proceed with the arguments of $\$ 6$ verbatim 2

Finally, there are two additional places that make some appeal to compactness. Theorem 7.2 uses compactness in order to show local uniform boundedness of the frame $e$ and to show integrability of $\left\|\partial_{s} \phi(s)\right\|_{L_{x}^{\infty}}$. In view of the result of $\sqrt{6}$, though, solutions are global, Schwartz, and with precompact image in $\mathcal{M}$ : therefore we may still use an explicit embedding, and all arguments carry over without modification. Theorem 7.6 employs compactness in a similar way; however, care must be taken if one desires to move from a local dynamic time interval to a global one. In particular, in order to attain uniform bounds, one must ensure that the particular dynamics in play keep $\phi$ within a bounded set.

\footnotetext{
${ }^{2}$ As an alternative, one may proceed with a more direct argument, only modifying the relevant parts of proofs as needed; the complete the details of this approach, however, seem to involve taking a long tour through the extensive literature on harmonic maps and harmonic map heat flow, most of which assumes the target manifold to be a closed manifold.
} 


\section{Appendix B. An ENERGy SPACE}

We propose an intrinsically defined energy space. As an application we have in view the study of the Schrödinger map initial value problem

$$
\begin{cases}\partial_{t} \phi & =J(\phi)\left(\phi^{*} \nabla\right)_{j} \partial_{j} \phi \\ \phi(0) & =\phi_{0}\end{cases}
$$

on a Kähler manifold $\mathcal{M}$ with complex structure $J$. See for instance [12] for definitions. Our approach is motivated by that pursued by Tao in [26] in the study of wave maps. The present setting is slightly simpler as compared to the wave maps setting in the sense that our Cauchy data includes only an initial position $\phi_{0}$ as opposed to both an initial position $\phi_{0}$ and initial velocity $\partial_{t} \phi_{0}$.

Let $\mathcal{L}$ denote the Hilbert space of pairs $\left(\psi_{s}, \psi_{x}\right)$ of measurable functions $\psi_{s}: \mathbf{R}^{+} \times \mathbf{R}^{2} \rightarrow \mathbf{R}^{m}$ and $\psi_{x}: \mathbf{R}^{+} \times \mathbf{R}^{2} \rightarrow \mathbf{R}^{m} \times \mathbf{R}^{m}$ whose norm

$$
\left\|\left(\psi_{s}, \psi_{x}\right)\right\|_{\mathcal{L}}:=\frac{1}{2} \int_{0}^{\infty} \int_{\mathbf{R}^{2}}\left|\psi_{s}\right|^{2} d x d s+\frac{1}{4} \int_{\mathbf{R}^{2}}\left|\psi_{x}\right|^{2} d x
$$

is finite. Note that the orthogonal group $S O(m)$ acts unitarily on $\mathbf{R}^{m}$ and so naturally on $\mathcal{L}$ unitarily. Quotienting out by this compact group, we obtain a metric space $S O(m) \backslash \mathcal{L}$.

Given classical initial data $\phi_{0}$ with energy $E_{0}<E_{\text {crit }}$, consider its heat flow extension $\phi(s, x)$ from $\mathbf{R}^{2}$ to $\mathbf{R}^{+} \times \mathbf{R}^{2}$. Choose a caloric gauge $e$ for $\phi_{0}$ that equals some fixed frame $e(\infty) \in \operatorname{Frame}\left(T_{\phi_{0}(\infty)} \mathcal{M}\right)$ at infinity. Let $S_{p}$ denote the set of functions $\mathbf{R}^{2} \rightarrow \mathcal{M}$ differing from $p \in \mathcal{M}$ by a Schwartz function. Hence $\phi_{0} \in S_{p}$ with $p:=\phi_{0}(\infty) \in \mathcal{M}$.

Define the nonlinear Littlewood-Paley resolution map $\jmath: \mathcal{S}_{p} \rightarrow S O(m) \backslash \mathcal{L}$ by

$$
\jmath\left(\phi_{0}\right):=S O(m)\left(\psi_{s}, \psi_{x}\right) .
$$

Rotating the frame $e(\infty)$ rotates the fields $\psi_{s}, \psi_{x}$ by an element of $S O(m)$, and thus the only arbitrary choice we have made is $p=\phi_{0}(\infty)$. We define the energy space $\dot{\mathcal{H}}_{p}^{1}$ to be the closure of $\jmath\left(\mathcal{S}_{p}\right)$ in $S O(m) \backslash \mathcal{L}$.

Lemma B.1. (Energy identity). For any $\phi \in \mathcal{S}_{p}$ we have

$$
E\left(\phi_{0}\right)=d_{S O(m) \backslash \mathcal{L}}\left(j\left(\phi_{0}\right), 0\right) .
$$

Proof. The left hand side of (B.2) may be written as

$$
\frac{1}{2} \int_{\mathbf{R}^{2}}\left|\psi_{x}(0, \cdot)\right|_{\phi_{0}^{*} h}^{2} d x
$$

Applying (7.4) yields

$$
\partial_{s}\left|\psi_{x}\right|^{2}=2 \psi_{j} \cdot D_{j} \psi_{s}
$$


and thus by integrating by parts and using (7.8) we obtain

$$
\begin{aligned}
\partial_{s} \frac{1}{2} \int_{\mathbf{R}^{2}}\left|\psi_{x}(s, \cdot)\right|^{2} d x & =-\int_{\mathbf{R}^{2}} D_{j} \psi_{j} \cdot \psi_{s} d x \\
& =-\int_{\mathbf{R}^{2}}\left|\psi_{s}\right|^{2} d x .
\end{aligned}
$$

As $E\left(\phi_{0}(s)\right) \rightarrow 0$ as $s \rightarrow \infty$ due to the energy monotonicity of the heat flow and the fact that $E_{0}<E_{\text {crit }}$, we conclude from (B.3) that

$$
E\left(\phi_{0}\right)=\frac{1}{2} \int_{0}^{\infty} \int_{\mathbf{R}^{2}}\left|\psi_{s}\right|^{2} d x d s+\frac{1}{4} \int_{\mathbf{R}^{2}}\left|\psi_{x}\right|^{2} d x,
$$

which, in view of (B.1), proves the lemma.

Theorem B.2. (Energy space). Let $p:=\phi_{0}(\infty) \in \mathcal{M}$ be fixed. Then there exists a complete metric space $\dot{\mathcal{H}}_{p}^{1}$ and a continuous map $\mathrm{\jmath}: \mathcal{S}_{p} \rightarrow \dot{\mathcal{H}}_{p}^{1}$ with the following properties:

(i) $\jmath\left(\mathcal{S}_{p}\right)$ is dense in $\dot{\mathcal{H}}_{p}^{1}$.

(ii) $\mathrm{J}$ is injective.

(iii) Translation and dilation on $\mathcal{S}_{p}$ extend to continuous isometric actions on $\dot{\mathcal{H}}_{p}^{1}$.

(iv) The energy functional $E: S_{p} \rightarrow \mathbf{R}^{+}$extends to a continuous functional $E: \dot{\mathcal{H}}_{p}^{1} \rightarrow \mathbf{R}^{+}$.

(v) If $\Phi \in \dot{\mathcal{H}}_{p}^{1}$ has energy $E(\Phi)=0$, then $\Phi=\jmath(p)$.

Proof. Property (i) follows from construction.

To show (ii), suppose we have classical data $\phi_{0}, \tilde{\phi}_{0} \in \mathcal{S}_{p}$ with $\jmath\left(\phi_{0}\right)=\jmath\left(\tilde{\phi}_{0}\right)$. Then there exist caloric gauges $e, \tilde{e}$ with respect to which $\psi_{s}=\tilde{\psi}_{s}$ and $\psi_{x}=\tilde{\psi}_{x}$. By construction $\phi_{0}(\infty)=p=\tilde{\phi}_{0}(\infty)$. If need be we apply a rotation in $S O(m)$ so that $e(\infty)=\tilde{e}(\infty)$. From (7.3) and (7.9) respectively we have that

$$
\partial_{s} \phi_{0}=e \psi_{s} \quad \text { and } \quad\left(\phi_{0} \nabla\right)_{s} e=0
$$

and hence a system of ODEs from which we may recover $\phi_{0}$ and $e$ from the boundary data $\phi_{0}(\infty)$ and $e(\infty)$, using (7.16) to justify integrability.

Property (iii) is an easy consequence of (i).

Property (iv) is also straightforward. We note, however, that as in [26] one may develop an energy space whose norm $\mathcal{L}$ is given by $\int_{0}^{\infty} \int_{\mathbf{R}^{2}}\left|\psi_{s}\right|^{2} d x d s$ rather than the one we adopted in (B.1). In such a case property (iv) requires verification and is not trivial. We do not pursue this approach here.

Property (v) follows from (i) and (iv). 


\section{REFERENCES}

[1] I. Bejenaru, A. D. Ionescu, C. E. Kenig, and D. Tataru, Global Schrödinger maps in dimensions $d \geq 2$ : small data in the critical Sobolev spaces, Ann. of Math. (2) $\mathbf{1 7 3}$ (2011), no. 3, 1443-1506. MR 2800718

[2] Yun Mei Chen, The weak solutions to the evolution problems of harmonic maps, Math. Z. 201 (1989), no. 1, 69-74. MR 990189 (90i:58030)

[3] Yun Mei Chen and Michael Struwe, Existence and partial regularity results for the heat flow for harmonic maps, Math. Z. 201 (1989), no. 1, 83-103. MR 990191 (90i:58031)

[4] J. Eells and J. C. Wood, Restrictions on harmonic maps of surfaces, Topology 15 (1976), no. 3, 263-266. MR 0420708 (54 \#8720)

[5] James Eells, Jr. and J. H. Sampson, Harmonic mappings of Riemannian manifolds, Amer. J. Math. 86 (1964), 109-160. MR 0164306 (29 \#1603)

[6] H. Koch and T. Lamm, Geometric flows with rough initial data, ArXiv e-prints: 0902.1488 (2009).

[7] Luc Lemaire, Applications harmoniques de surfaces riemanniennes, J. Differential Geom. 13 (1978), no. 1, 51-78. MR 520601 (80h:58024)

[8] Peter Li and Luen-Fai Tam, The heat equation and harmonic maps of complete manifolds, Invent. Math. 105 (1991), no. 1, 1-46. MR 1109619 (93e:58039)

[9] Fang-Hua Lin, Gradient estimates and blow-up analysis for stationary harmonic maps, Ann. of Math. (2) 149 (1999), no. 3, 785-829. MR 1709303 (2000j:58028)

[10] FangHua Lin and ChangYou Wang, Harmonic and quasi-harmonic spheres, Comm. Anal. Geom. 7 (1999), no. 2, 397-429. MR 1685578 (2000b:58028)

[11] Fanghua Lin and Changyou Wang, The analysis of harmonic maps and their heat flows, World Scientific Publishing Co. Pte. Ltd., Hackensack, NJ, 2008. MR 2431658 (2011a:58030)

[12] Helena McGahagan, An approximation scheme for Schrödinger maps, Comm. Partial Differential Equations 32 (2007), no. 1-3, 375-400. MR 2304153 (2009e:35265)

[13] Jean-Claude Mitteau, Sur les applications harmoniques, J. Differential Geometry 9 (1974), 41-54. MR 0345129 (49 \#9868)

[14] Charles B. Morrey, Jr., Multiple integrals in the calculus of variations, Classics in Mathematics, Springer-Verlag, Berlin, 2008, Reprint of the 1966 edition [MR0202511]. MR 2492985

[15] Peter Petersen, Riemannian geometry, Graduate Texts in Mathematics, vol. 171, Springer-Verlag, New York, 1998. MR 1480173 (98m:53001)

[16] Richard Schoen and Karen Uhlenbeck, A regularity theory for harmonic maps, J. Differential Geom. 17 (1982), no. 2, 307-335. MR 664498 (84b:58037a)

[17] Paul Smith, Conditional global regularity of Schrödinger maps: sub-threshold dispersed energy, ArXiv e-prints: 1012.4048 (2010).

[18] _ Global regularity of critical Schrödinger maps: subthreshold dispersed energy, ArXiv e-prints: 1112.0251 (2011).

[19] Michael Spivak, A comprehensive introduction to differential geometry. Vol. II, second ed., Publish or Perish Inc., Wilmington, Del., 1979. MR 532831 (82g:53003b)

[20] Michael Struwe, On the evolution of harmonic mappings of Riemannian surfaces, Comment. Math. Helv. 60 (1985), no. 4, 558-581. MR 826871 (87e:58056)

[21] _ On the evolution of harmonic maps in higher dimensions, J. Differential Geom. 28 (1988), no. 3, 485-502. MR 965226 (90j:58037)

[22] Terence Tao, Gauges for the Schrödinger map.

[23] _ Geometric renormalization of large energy wave maps, Journées "Équations aux Dérivées Partielles", École Polytech., Palaiseau, 2004, pp. Exp. No. XI, 32. MR 2135366 (2006i:58044) 
[24] - Nonlinear dispersive equations, CBMS Regional Conference Series in Mathematics, vol. 106, Published for the Conference Board of the Mathematical Sciences, Washington, DC, 2006, Local and global analysis. MR 2233925 (2008i:35211)

[25] Global regularity of wave maps III. Large energy from $\boldsymbol{R}^{1+2}$ to hyperbolic spaces, ArXiv e-prints: 0805.4666 (2008).

[26] _ Global regularity of wave maps IV. Absence of stationary or self-similar solutions in the energy class, ArXiv e-prints: 0806.3592 (2008).

[27] Global regularity of wave maps VII. Control of delocalised or dispersed solutions, ArXiv e-prints: 0908.0776 (2009).

[28] Peter Topping, The optimal constant in Wente's $L^{\infty}$ estimate, Comment. Math. Helv. 72 (1997), no. 2, 316-328. MR 1470094 (98k:35075)

[29] Reverse bubbling and nonuniqueness in the harmonic map flow, Int. Math. Res. Not. (2002), no. 10, 505-520. MR 1883901 (2002k:53132)

[30] _ Bubbling of almost-harmonic maps between 2-spheres at points of zero energy density, Variational problems in Riemannian geometry, Progr. Nonlinear Differential Equations Appl., vol. 59, Birkhäuser, Basel, 2004, pp. 33-42. MR 2076265 (2005d:58029)

[31] - Improved regularity of harmonic map flows with Hölder continuous energy, Calc. Var. Partial Differential Equations 21 (2004), no. 1, 47-55. MR 2078746 (2005c:53088)

[32] _ Repulsion and quantization in almost-harmonic maps, and asymptotics of the harmonic map flow, Ann. of Math. (2) 159 (2004), no. 2, 465-534. MR 2081434 (2005g:58029)

[33] Winding behaviour of finite-time singularities of the harmonic map heat flow, Math. Z. 247 (2004), no. 2, 279-302. MR 2064053 (2004m:53120)

[34] Hans Triebel, Theory of function spaces. II, Monographs in Mathematics, vol. 84, Birkhäuser Verlag, Basel, 1992. MR 1163193 (93f:46029)

\section{University of CALifornia, Berkeley}

E-mail address: smith@math.berkeley.edu 